\title{
An SINR-Aware Joint Mode Selection, Scheduling, and Resource Allocation Scheme for D2D Communications
}

Bithas, Petros S.; Maliatsos, Konstantinos; Foukalas, Fotis

Published in:

IEEE Transactions on Vehicular Technology

Link to article, DOI:

10.1109/TVT.2019.2900176

Publication date:

2019

Document Version

Peer reviewed version

Link back to DTU Orbit

Citation $(A P A)$ :

Bithas, P. S., Maliatsos, K., \& Foukalas, F. (2019). An SINR-Aware Joint Mode Selection, Scheduling, and Resource Allocation Scheme for D2D Communications. IEEE Transactions on Vehicular Technology, 68(5), 4949-4963. https://doi.org/10.1109/TVT.2019.2900176

\section{General rights}

Copyright and moral rights for the publications made accessible in the public portal are retained by the authors and/or other copyright owners and it is a condition of accessing publications that users recognise and abide by the legal requirements associated with these rights.

- Users may download and print one copy of any publication from the public portal for the purpose of private study or research.

- You may not further distribute the material or use it for any profit-making activity or commercial gain

- You may freely distribute the URL identifying the publication in the public portal 


\title{
An SINR-Aware Joint Mode Selection, Scheduling, and Resource Allocation Scheme for D2D Communications
}

\author{
Petros S. Bithas, Member, IEEE, Konstantinos Maliatsos, and Fotis Foukalas, Member, IEEE
}

\begin{abstract}
In this paper, a new mode selection (MS) scheme for device-to-device (D2D) enabled cellular networks is proposed. The MS is carried out using a predefined signal-to-interference plus noise ratio threshold and it aims to guarantee a minimum level of quality of service. With the new scheme, interfering effects are taken into consideration, while realistic assumptions for the acquisition of the channel information are made. Assuming a single-user case, an analytical framework is developed, which is based on the Markov-chain theory, and is used to study the system's performance. The various performance results that have been obtained reveal that with the proposed MS, the probability of mode switching can be reduced (as compared to other systems) and thus, an increase on the overhead and signal processing is avoided. Moreover, the new approach is applied in a multi-user communication scenario, where a joint MS, resource allocation (RA), and scheduling optimization problem is formulated. The solution to this problem is based on a greedy heuristic algorithm that properly assigns the available resources among cellular users and D2D pairs. The joint MS, RA, and scheduling algorithm considers limited SINR awareness and it provides significant performance improvement in terms of sum-rate.
\end{abstract}

Index Terms-Device-to-device communications, Markov chain, multi-user, performance analysis, resource allocation, scheduling, SINR-aware mode selection, sum rate.

\section{INTRODUCTION}

D EVICE-to-device (D2D) communications have attracted considerable attention by the scientific community, the industry, and the standardization bodies over the past several years [1]. Reasons for this include the improved system throughput and spectral efficiency that is offered, the extension of the network coverage and the battery lifetime of the user equipment (UE), e.g., [2], [3]. Depending on the spectrum that is used for realizing D2D communications, different cases have been studied such as the in-band, when D2D UEs use the same frequency spectrum with the cellular ones, and the out-of-band, when different bands are used by the D2D UEs. Focusing on the in-band scenario, several resource allocation (RA) modes have been proposed, including the underlay and

Manuscript received January 14, 2018; revised July 7, 2018 and November 13; accepted December 25, 2018. Date of publication XXXXXX XX, 2019; date of current version XXXXX XX, 2019. This work has been supported by the "Spectrum Overlay through Aggregation of Heterogeneous Dispersed Bands" project, ICT-SOLDER, www.ict-solder.eu, FP7 grant agreement number 619687.

P. S. Bithas is with the General Department, National and Kapodistrian University of Athens, Greece (e-mail: pbithas@ ieee.org).

K. Maliatsos is with both the Department of Digital Systems, University of Piraeus, Greece and the FERON technologies (e-mail: kmaliat@unipi.gr).

F. Foukalas is with the IoT Research Center at Technical University of Denmark (e-mail: foukalas@ieee.org). the overlay [4], [5]. In the underlay mode, the D2D pairs and the cellular UEs (CUEs) share the same resources, an approach that offers improved spectral efficiency with the cost of increased levels of interference. In the overlay mode, the D2D pairs utilize dedicated resources, an approach that offers reduced interference, with the cost of spectrum underutilization. However, under the assumption of un-coordinated communications, interfering effects coming from other D2D pairs and/or UEs belonging to nearby cells, are always present, which complicates the decision for the mode selection (MS).

MS refers to the mechanism used for selecting D2D or cellular mode of operation. Several approaches have been proposed for MS, e.g., [3], [6]-[13]. In [6], a new interference management scheme is proposed to improve the reliability of D2D communication in the uplink (UL), by exploiting the retransmission of the interference from the eNodeB (eNB). In [8], an analytical framework is presented for enabling D2D communications based on a flexible MS along with truncated channel inversion power control. In [3], a new threshold-based MS is proposed in a D2D enabled cellular network, which exploits the received signal strength. For this new scheme, an analytical framework is developed for investigating the downlink outage probability (OP), assuming Rayleigh fading. In [12], a centralized opportunistic access control scheme and a MS mechanism are proposed that reduce cross-tier interferences and improve system's performance. Finally, in [13], a mode selection scheme, based on a threshold is proposed, which however is related with the received signal strength and not the received signal-to-interference plus noise ratio (SINR).

In most multi-user (MU) scenarios, MS is tackled jointly with RA and scheduling for both operation modes. Joint MS/RA is necessary in order to control interference levels and achieve performance improvement. In [14], underlaying resource sharing between cellular and D2D systems is investigated under practical constraints such as minimum and maximum spectral efficiency. A sum-rate improvement method using evolutionary algorithm is proposed in [15]. An exhaustive strategy for a similar system model is presented in [16], while distributed power control for the coexistence of a cellular network with underlaying D2D links is presented in [17]. A greedy heuristic algorithm is also proposed in [18] for reusing the downlink (DL) and UL resources of long term evolution (LTE) networks. In [19] and [20], three operation modes are identified: i) cellular, ii) dedicated (no reuse of cellular resources by devices is allowed), and iii) reuse (underlaying), providing sum-rate maximization schemes for all modes. The 
work of [21] focuses on cellular/dedicated modes and proposes an opportunistic scheduling scheme with quality-of-service (QoS) requirement constraints. In [22], the joint issue of MS, UL reusing allocation, and power management is addressed based on a coalition formation game for mobile D2D communications. In [23], joint MS, channel assignment, and power control in D2D communications is addressed, which aims at maximizing the overall system throughput while guaranteeing the SINR of both D2D and cellular links.

A general observation in the above literature review is that the eNB is assumed to have knowledge of all information (regarding the channel conditions among the network elements) for performing the MS, while the mode of operation is selected without considering the interference effects. In this paper, inspired by the channel selection technique proposed in [24], an alternative MS scheme is proposed, which (i) exploits the received SINR and (ii) is based on realistic assumptions for the acquisition of the channel information. Another important advantage of the new scheme is that it offers improved performance with relative low complexity, as compared to other approaches. The new scheme is applied in two communication scenarios, namely in a single-user (SU) one, where an analytical framework is deduced and in a MU one where a joint MS, RA, and scheduling optimization framework is proposed. More specifically, the contribution of this work is summarized as follows:

- For the proposed MS scheme, a stochastic analysis for the received SINR is presented that employs a finitestate Markov chain. To this end, closed-form general expressions are derived for the transition and stationary probabilities of the Markov chain;

- Generic statistical characteristics are derived for the UE received SINR, such as the probability density function (PDF) and the cumulative distribution function (CDF);

- Simplified expressions are also derived assuming signal to interference ratio (SIR) statistics as well as for higher values of the average signal-to-noise ratio (SNR) that facilitate the performance analysis;

- Closed-form expressions for important performance metrics of the considered systems are provided, such as the $\mathrm{OP}$, the bit error probability (BEP), the capacity, while a complexity analysis has been also included;

- The new scheme is also used to develop a joint MS, RA, and scheduling algorithm, where it is assumed that i) the eNB has not a complete knowledge of the radio network status, ii) limited feedback from the devices to the eNB is available. The scheme supports conventional and D2D communications and it is applied on the UL resources;

- A new low complexity greedy heuristic algorithm is proposed for solving the optimization problem defined in the joint MS, RA, and scheduling algorithm;

- The later one is compliant with the latest releases of LTE that support D2D communications [25];

- The performance of the joint MS, RA, and scheduling algorithm with limited SINR-awareness is evaluated through simulation, verifying significant improvement in terms of sum-rate and UE SINR;
The remainder of this paper is organized as follows. A general description of the system and channel models is presented in Section II. In Section III, the proposed MS scheme is presented and in Section IV its performance is analysed for the SU case. In Section V, the joint MS/RA framework is presented, while in Section VI its performance is evaluated based on simulated results. Finally, the conclusions of this work can be found in Section VII. For the reader's convenience, most of the notations and symbols used in the rest of the paper are summarized in Table I.

\section{System AND ChANNEL Model}

\section{A. System Model}

A D2D-enabled cellular network is assumed, where two types of UEs exist: the cellular UEs (CUEs) and the device UEs (DUEs). Two operation modes may be supported by the UEs: Mode 1, D2D operation and Mode 2, Cellular operation. The CUEs are able to operate only in the conventional cellular mode (Mode 2), while the DUEs are able to use both modes. In order to be compliant with the latest LTE release [25], it is assumed that D2D links, also referred as sidelinks (SLs), are established on the UL resources of the cellular network. Moreover, it is assumed that all UE communication links are suffering from interference. The system model under consideration is presented in Fig. 1.

Let $\mathcal{C}=\left\{C_{l} \mid l=1,2, \ldots, L_{C}\right\}$ be the CUE set, where $L_{C}$ is the number of active CUEs in the network. Moreover, $\mathcal{D}=$ $\left\{D_{j} \mid j=1,2, \ldots, L_{D}\right\}$ is the set with the DUEs, while $L_{D}$ is the number of possible D2D pairs. The DUEs are organized in pairs, where a given transmitter (Tx) DUE denoted as $D_{j}^{T x}$ attempts direct communication with a corresponding receiver (Rx) DUE $D_{j}^{R x}$ forming the D2D pair $D_{j}$. Since, DUEs are able to select either modes of operation, some D2D pairs may be established through the cellular network. Set $\mathcal{D}_{1}$ includes the pairs in D2D mode (Mode 1), while set $\mathcal{D}_{2}$ includes the pairs in cellular mode (Mode 2), i.e., $\mathcal{D}=\mathcal{D}_{1} \cup \mathcal{D}_{2}$. The set of all UEs operating in cellular mode is given by $\mathcal{G}=\mathcal{C} \cup \mathcal{D}_{2}$.

Mode selection is assumed to be performed periodically, jointly with the RA and scheduling procedures with $T_{r r m}$ period. The following assumptions are used:

- eNB has no direct knowledge of the SINRs for the links between device pairs, since the eNB is not able to measure them;

- Any information for D2D SINRs used for MS, RA, and scheduling is acquired through a D2D SL channel state reporting procedure that increases the signaling overhead for the D2D operation mode;

- Devices know the SINR of their D2D link;

- Devices are able to measure the received signal power from all adjacent devices that perform SL transmission;

- The SL request is initiated by the Rx node of the D2D pair and the D2D Tx is obliged to follow;

- The BS has the right to deny the D2D SL request based on the results of the RA and scheduling algorithm;

- Single antenna Tx and Rx are considered for all network elements;

- Quasi-stationary flat fading channels are assumed. 


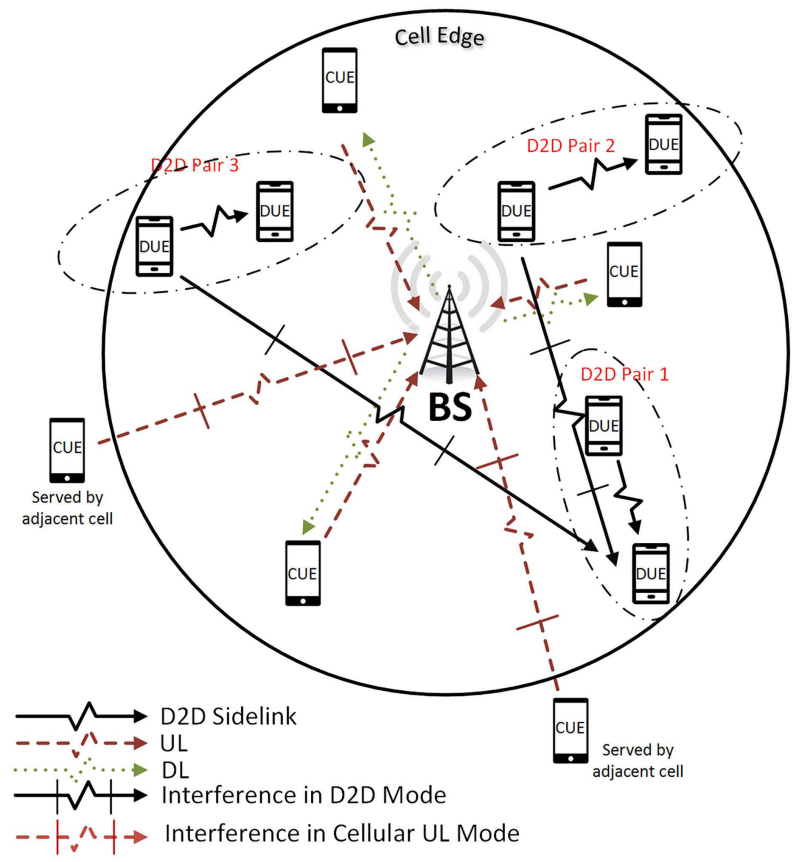

Fig. 1: System model: UEs operating in Cellular or D2D mode and respective interference sources.

\section{B. Channel Model}

For a period of $T_{r r m}$, flat fading links per subchannel and independent block fading conditions are assumed, similar to many works, e.g., [26]. For a given UE, regardless of the operation mode $i$ (with $i \in\{1,2\}$ ) and assuming $L$ interfering signals, the received SINR can be expressed as

$$
\gamma_{i}=\frac{P_{d}\left|\beta_{(X, Y)}^{(k)}\right|^{2} d_{(X, Y)}^{-\alpha}}{P_{i} \sum_{j_{i}=1}^{L}\left|\beta_{\left(X_{j_{i}}, Y\right)}^{(k)}\right|^{2} d_{\left(X_{j_{i}}, Y\right)}^{-\alpha}+N_{0}},
$$

where $P_{d}$ denotes the desired transmit power, $P_{i}$ the (equal) transmit power of the interfering sources, $N_{0}$ the noise power, $\alpha$ the path loss exponent, and $d_{(X, Y)},\left|\beta_{(X, Y)}\right|$ the distance and the Rayleigh faded coefficient between nodes $X$ and $Y$, respectively. It is noted that for both modes of operation, the interfering effects are due to other DUEs, from the same or other cells as well as from cellular users belonging to other cells, as it is also shown in Fig. 1. In this context, the following closed-form expression for the PDF of $\gamma_{i}$ can be obtained

$$
f_{\gamma_{i}}(\gamma)=\frac{\exp \left(-\frac{\gamma}{\bar{\gamma}_{i}}\right)}{\bar{\gamma}_{i}} \mathcal{S}_{i}\left[\frac{1}{\left(\frac{\gamma}{\bar{\gamma}_{i}}+\frac{1}{\bar{\gamma}_{I_{j_{i}}, \bar{i}}}\right)^{2}}+\frac{1}{\frac{\gamma}{\bar{\gamma}_{i}}+\frac{1}{\bar{\gamma}_{I_{j_{i}}, \bar{i}}}}\right],
$$

where

$$
\mathcal{S}_{i}=\sum_{j_{i}=1}^{L}\left(\prod_{j_{i}=1}^{L} \frac{1}{\bar{\gamma}_{I_{j_{i}}, \bar{i}}}\right) \frac{1}{\prod_{\substack{m=1 \\ m \neq j_{i}}}^{L} \frac{1}{\bar{\gamma}_{I_{m}, \bar{i}}}-\frac{1}{\bar{\gamma}_{I_{j_{i}}, \bar{i}}}},
$$

\begin{tabular}{|c|c|}
\hline$\overline{\mathcal{C}}$ & Set of cellular UEs \\
\hline$L_{C}$ & Number of active CUEs in the network \\
\hline$\overline{L_{D}}$ & Number of possible D2D pairs \\
\hline$\overline{\mathcal{D}_{1}}$ & Pairs in the D2D mode \\
\hline $\mathcal{D}_{2}$ & Pairs in the cellular mode \\
\hline $\mathcal{D}$ & $\mathcal{D}_{1} \cup \mathcal{D}_{2}$ \\
\hline $\mathcal{G}$ & $\mathcal{C} \cup \mathcal{D}_{2}$ \\
\hline$P P_{d}$ & Transmit power of the desired signal \\
\hline$P_{i}$ & Transmit power of the interfering sources \\
\hline$N_{0}$ & Noise power \\
\hline$d_{(X, Y)}$ & Distance between nodes $X$ and $Y$ \\
\hline$\left|\beta_{(X, Y)}\right|$ & Channel fading coefficient between $X$ and $Y$ \\
\hline $\bar{\gamma}_{i}$ & $P_{t} d_{X, Y}^{-\alpha}$ \\
\hline$P_{t}$ & $P_{d} / N_{0}$ \\
\hline$\overline{\bar{\gamma}}_{x, y}$ & $P_{I} d_{X, Y}^{-\alpha}$ \\
\hline$P P_{I}$ & $P_{i} / N_{0}$ \\
\hline$i$ & $3-i$ \\
\hline$\gamma_{\mathrm{th}}$ & Threshold for switching operation (SO) \\
\hline$A_{i_{n}}$ & $\frac{(-1)^{n+1}}{\Lambda_{1}}$, with $n \in\{1,2\}, \Lambda_{1}=\frac{\bar{\gamma}_{i}}{\bar{\gamma}_{I_{j_{1}}, \bar{i}}}-\frac{\bar{\gamma}_{\bar{i}}}{\bar{\gamma}_{I_{j_{2}}, i}}$ \\
\hline$a_{1_{1}}$ & $\frac{\overline{\gamma_{\bar{i}}}}{\overline{\bar{\gamma}_{I_{\bar{j}}, i}}}$ \\
\hline$a_{1_{2}}$ & $\frac{\overline{\bar{\gamma}_{i}}}{\bar{\gamma}_{I_{j,}, \bar{i}}}$ \\
\hline$b_{n_{m}}$ & $(n, m) \in\{1,2\}$ \\
\hline$A_{2_{n}}$ & $\frac{(-1)^{n+1}}{\Lambda_{2}^{2}}$, with $\Lambda_{2}=\frac{\bar{\gamma}_{\bar{i}}}{\bar{\gamma}_{I_{j}, i}}-\frac{\bar{\gamma}_{i}}{\bar{\gamma}_{I_{j_{1}, i}}}$ \\
\hline$A_{2_{3}}$ & $\begin{array}{lllllll} & J_{2} & J_{1}\end{array}$ \\
\hline$a_{2_{1}}$ & $\frac{\overline{\bar{\gamma}_{\bar{i}}}}{\bar{\gamma}_{I_{j_{2}}, i}}$ \\
\hline$a_{2_{m}}$ & $\frac{\frac{J 2}{\bar{\gamma}_{i}}}{\overline{\bar{\gamma}_{I_{j_{1}}, \bar{i}}}}$ with $m \in\{2,3\}$ \\
\hline$b_{2_{3}}$ & 2 \\
\hline $\bar{\gamma}$ & $\frac{\overline{\bar{\gamma}_{i}+}+\bar{\gamma}_{i}}{\bar{\gamma}_{\bar{j}} \bar{\gamma}_{i}}$ \\
\hline$a_{1_{3}}$ & $\bar{\gamma}_{i} / \bar{\gamma}_{\bar{i}}$ \\
\hline$\gamma_{T}$ & Outage threshold \\
\hline
\end{tabular}

TABLE I: Notations AND Symbols

$\bar{\gamma}_{i}=P_{t} d_{X, Y}^{-\alpha}$ denotes the average received SNR (with $P_{t}=$ $\left.P_{d} / N_{0}\right), \bar{\gamma}_{x, y}=P_{I} d_{X, Y}^{-\alpha}$ denotes the average INR, i.e., a virtual way to describe the received SNR of the interfering signals (with $P_{I}=P_{i} / N_{0}$ ), and $\bar{i}=3-i$.

\section{SinR-Aware Mode Selection: A Markov Chain APPROACH}

The proposed MS criterion exploits the received SINR. In particular, in each $T_{r r m}$ period, it is examined whether the SINR of the previously selected operation mode, $\gamma_{i}(n-1)$, exceeds a predefined threshold $\gamma_{\text {th }}$. In that case, the same mode is selected for the current period, otherwise it switches to the mode having the highest instantaneous SINR. Based on this approach, the number of mode SOs will be reduced, since depending upon the value of $\gamma_{\mathrm{th}}$, the received SINR of the previous mode can be also satisfactory at the current period, avoiding thus unnecessary SO. A reduced number of SOs will also result to an important reduction on the information that should be exchanged among the network elements. 
Assuming slow and independent fading between $T_{r r m}$, the proposed selection scheme defines a 2-state ergodic Markov chain that is also regular, where the $i$-th state corresponds to the event that mode $i$ is selected. Therefore, it is obvious that MS depends on the current selected mode (state) as well as the fading behavior of the desired and interfering links. This Markov chain will be characterized by a unique stable vector of stationary probabilities $\pi$. This results to a transition matrix $P$ that has only positive entries (regularity) and thus it is possible to switch from the one mode of operation to the other (ergodicity). Based on the proposed MS scheme, the transition probabilities of the corresponding Markov chain are given as follows

$$
P_{i, j}=\left\{\begin{array}{c}
\operatorname{Pr}\left[\gamma_{i} \geq \gamma_{\mathrm{th}}\right]+\operatorname{Pr}\left[\gamma_{i}<\gamma_{\mathrm{th}}, \gamma_{i} \geq \gamma_{j}\right], i=j \\
\operatorname{Pr}\left[\gamma_{i}<\gamma_{\mathrm{th}}, \gamma_{j} \geq \gamma_{i}\right], \quad i \neq j .
\end{array}\right.
$$

In $(3)$, it is clear that

$$
\operatorname{Pr}\left[\gamma_{i} \geq \gamma_{\mathrm{th}}\right]=1-F_{\gamma_{i}}\left(\gamma_{\mathrm{th}}\right)
$$

where $F_{\gamma_{i}}\left(\gamma_{\mathrm{th}}\right)$ can directly be evaluated using (2) as

$$
F_{\gamma_{i}}\left(\gamma_{\mathrm{th}}\right)=1-\mathcal{S}_{i} \frac{\bar{\gamma}_{i} \bar{\gamma}_{j_{i}, \bar{i}}}{\bar{\gamma}_{i}+\gamma_{\mathrm{th}} \bar{\gamma}_{j_{i}, \bar{i}}} \exp \left(-\frac{\gamma_{\mathrm{th}}}{\bar{\gamma}_{i}}\right) .
$$

Moreover, as shown in Appendix A, the probability defined in (3), i.e., $\operatorname{Pr}\left[\gamma_{i}<\gamma_{\text {th }}, \gamma_{i} \geq \gamma_{j}\right]$, has been evaluated as

$$
\begin{aligned}
& \operatorname{Pr}\left[\gamma_{i}<\gamma_{\mathrm{th}}, \gamma_{i} \geq \gamma_{\bar{i}}\right]=\mathcal{S}_{1} \mathcal{S}_{2}\left[\bar{\gamma}_{I_{j_{1}}, \bar{i}} \bar{\gamma}_{I_{j_{2}}, i}\right. \\
& \left.\times\left(1-\frac{\exp \left(-\frac{\gamma_{\mathrm{th}}}{\bar{\gamma}_{i}}\right) \bar{\gamma}_{i}}{\bar{\gamma}_{i}+\gamma_{\mathrm{th}} \bar{\gamma}_{I_{j_{i}}, \bar{i}}}\right)-\bar{\gamma}_{\bar{i}}\left(\mathcal{H}(1)+\bar{\gamma}_{i} \mathcal{H}(2)\right)\right] .
\end{aligned}
$$

In (6)

$$
\begin{aligned}
& \mathcal{H}(x)=\sum_{\ell=1}^{x+1} A_{x_{1}} \exp \left(\frac{a_{x_{\ell}}}{\bar{\gamma}}\right)\left[\operatorname{Ei}\left(b_{x_{\ell}}, \frac{a_{x_{\ell}}}{\bar{\gamma}}\right) a_{x_{\ell}}^{1-b_{x_{\ell}}}\right. \\
& \left.-\operatorname{Ei}\left(b_{x_{\ell}}, \frac{a_{x_{\ell}}+\gamma_{\mathrm{th}}}{\bar{\gamma}}\right)\left(a_{x_{\ell}}+\gamma_{\mathrm{th}}\right)^{1-b_{x_{\ell}}}\right],
\end{aligned}
$$

where $\operatorname{Ei}(\cdot)$ denotes the exponential integral [27, eq. (8.21/1)]. Moreover, for $i \neq j, P_{i, j}=1-P_{i, i}$. Using the transition probabilities presented in (6), the limiting probability are given by $\pi_{1}=\frac{P_{2,1}}{1-P_{1,1}+P_{2,1}}$ and $\pi_{2}=\frac{1-P_{1,1}}{1-P_{1,1}+P_{2,1}}$. Based on them, the CDF of the output SINR of the proposed scheme, $\gamma_{\text {out }}$, is given by

$$
F_{\gamma_{\text {out }}}(\gamma)=\left\{\begin{array}{c}
\sum_{i=1}^{2} \pi_{i}\left\{F_{\gamma_{i}}(\gamma)-F_{\gamma_{i}}\left(\gamma_{\mathrm{th}}\right)\right. \\
\left.+F_{\gamma_{i}}\left(\gamma_{\mathrm{th}}\right) F_{\gamma_{\bar{i}}}(\gamma)\right\}, \gamma \geq \gamma_{\mathrm{th}} \\
F_{\gamma_{1}}(\gamma) F_{\gamma_{2}}(\gamma), \quad \gamma<\gamma_{\mathrm{th}}
\end{array}\right.
$$

Differentiating (7) with respect to $\gamma$, yields the corresponding expression for the PDF of $\gamma_{\text {out }}$ as

$$
f_{\gamma_{\text {out }}}(\gamma)=\left\{\begin{array}{c}
\sum_{i=1}^{2} \pi_{i}\left\{f_{\gamma_{i}}(\gamma)+F_{\gamma_{i}}\left(\gamma_{\mathrm{th}}\right) f_{\gamma_{\bar{i}}}(\gamma)\right\}, \gamma \geq \gamma_{\mathrm{th}} \\
\sum_{i=1}^{2} f_{\gamma_{i}}(\gamma) F_{\gamma_{\bar{i}}}(\gamma), \quad \gamma<\gamma_{\mathrm{th}} .
\end{array}\right.
$$

Next, two special cases will be analytically studied, namely an interference limited scenario and a high SNR scenario. Both these results, will be used in the next sections for further analytical investigations.

\section{A. Special Case 1: Signal-to-Interference Ratio Statistics}

In many cases, the wireless communication systems tend to be interference limited rather than noise limited, since the thermal and man-made noise effects are often insignificant compared to the signal levels of co-channel users [3]. Thus considering an interference limited environment, i.e., ignoring the AWGN at UEs, the received SIR is given by

$$
\gamma_{i}=\frac{P_{t}\left|\beta_{(X, Y)}^{(k)}\right|^{2} d_{(X, Y)}^{-\alpha}}{P_{I} \sum_{j_{i}=1}^{L}\left|\beta_{\left(X_{j_{i}}, Y\right)}^{(k)}\right|^{2} d_{\left(X_{j_{i}}, Y\right)}^{-\alpha}} .
$$

In this case, the PDF of $\gamma_{i}$ can be obtained as

$$
f_{\gamma_{i}}(\gamma)=\frac{1}{\bar{\gamma}_{i}} \mathcal{S}_{i}\left[\frac{1}{\left(\frac{\gamma}{\bar{\gamma}_{i}}+\frac{1}{\bar{\gamma}_{I_{j}, i}}\right)^{2}}\right]
$$

and the corresponding CDF expression is given by

$$
F_{\gamma_{i}}(\gamma)=\mathcal{S}_{i}\left[\frac{1}{\left(\frac{\gamma}{\bar{\gamma}_{i}}+\frac{1}{\bar{\gamma}_{I_{j}, \bar{i}}}\right)^{2}}\right] \frac{\gamma \bar{\gamma}_{I_{j_{i}}, \bar{i}}^{2}}{\bar{\gamma}_{i}+\gamma \bar{\gamma}_{I_{j_{i}}, \bar{i}}}
$$

Based on (10), following a similar approach as the one used for deriving (6), and using [28, eqs. (1.2.11/5) and (1.2.10/16)], the following closed-form expression for $\operatorname{Pr}\left[\gamma_{i}<\gamma_{\mathrm{th}}, \gamma_{i} \geq \gamma_{\bar{i}}\right]$ is derived

$$
\begin{aligned}
& \operatorname{Pr}\left[\gamma_{i}<\gamma_{\mathrm{th}}, \gamma_{i} \geq \gamma_{\bar{i}}\right]=1-F_{\gamma_{i}}\left(\gamma_{\mathrm{th}}\right)+\mathcal{S}_{1} \mathcal{S}_{2}\left\{\overline { \gamma } _ { I _ { j _ { i } } , \overline { i } } ^ { 2 } \left[\bar{\gamma}_{i}\right.\right. \\
& {\left[\ln \left(a_{1_{3}}\right)+\ln \left(\frac{\bar{\gamma}_{\bar{i}}+\gamma_{\mathrm{th}} \bar{\gamma}_{I_{j_{i}}, \bar{i}}}{\bar{\gamma}_{i}+\gamma_{\mathrm{th}} \bar{\gamma}_{I_{j_{\bar{i}}}, i}}\right)\right]+\gamma_{\mathrm{th}} \bar{\gamma}_{I_{j_{\bar{i}}}, i}+a_{1_{3}}} \\
& \left.\left.\times \gamma_{\mathrm{th}} \bar{\gamma}_{I_{j_{i}}, \bar{i}}\left(\ln \left(\frac{\bar{\gamma}_{\bar{i}}+\gamma_{\mathrm{th}} \bar{\gamma}_{I_{j_{i}}, \bar{i}}}{\bar{\gamma}_{i}+\gamma_{\mathrm{th}} \bar{\gamma}_{I_{j_{\bar{i}}}, i}}\right)+\ln \left(a_{1_{3}}\right)-1\right)\right]\right\} \\
& \times \frac{1}{\left(\bar{\gamma}_{i}+\gamma_{\mathrm{th}} \bar{\gamma}_{I_{j_{1}, \bar{i}}}\right)\left(1-\frac{a_{1_{2}}}{a_{1_{1}}}\right)^{2}},
\end{aligned}
$$

where $a_{1_{3}}=\bar{\gamma}_{i} / \bar{\gamma}_{\bar{i}}$. Also in this case, for $i \neq j, P_{i, j}=1-P_{i, i}$. Using the transitions probabilities, the limiting probabilities can be evaluated and based on them the PDF and CDF expressions can be directly evaluated using (10) and (11) in (8) and (7), respectively.

\section{B. Special Case 2: High SNR Analysis}

At the high SNR regime and due to the Taylor's series expansion of the exponential function, the exponential PDF and CDF expressions can be closely approximated by $f_{\gamma_{i}}(\gamma) \cong 1 / \bar{\gamma}_{i}$ and $F_{\gamma_{i}}(\gamma) \cong \gamma / \bar{\gamma}_{i}$, respectively. Based 
on these approximated expressions, the following convenient closed-form expression for the PDF of $\gamma_{i}$ is derived

$$
f_{\gamma_{i}}(\gamma) \cong \mathcal{S}_{i} \frac{\bar{\gamma}_{I_{j_{i}}, \bar{i}}+\bar{\gamma}_{I_{j_{i}}, \bar{i}}^{2}}{\bar{\gamma}_{i}} \exp \left(-\frac{\gamma}{\bar{\gamma}_{i}}\right),
$$

with the corresponding CDF expression given by

$$
F_{\gamma_{i}}(\gamma) \cong \mathcal{S}_{i}\left(\bar{\gamma}_{I_{j_{i}}, \bar{i}}+\bar{\gamma}_{I_{j_{i}}, \bar{i}}^{2}\right)\left[1-\exp \left(-\frac{\gamma}{\bar{\gamma}_{i}}\right)\right] .
$$

Based on (13) and on the approach used for deriving (6), the following closed-form expression for $\operatorname{Pr}\left[\gamma_{i}<\gamma_{\text {th }}, \gamma_{i} \geq \gamma_{\bar{i}}\right]$ is derived

$$
\begin{aligned}
& \operatorname{Pr}\left[\gamma_{i}<\gamma_{\mathrm{th}}, \gamma_{i} \geq \gamma_{\bar{i}}\right] \cong 1-F_{\gamma_{i}}\left(\gamma_{\mathrm{th}}\right)+\mathcal{S}_{1} \mathcal{S}_{2} \\
& \times \prod_{p=1}^{2}\left(\bar{\gamma}_{I_{j_{p}}, p}+\bar{\gamma}_{I_{j_{p}}, p}^{2}\right)\left[F_{\gamma_{i}}\left(\gamma_{\mathrm{th}}\right)-\frac{\bar{\gamma}_{\bar{i}}}{\bar{\gamma}_{i}+\bar{\gamma}_{\bar{i}}}\right. \\
& \left.\times\left(1-\exp \left(-\left(\frac{1}{\bar{\gamma}_{i}}+\frac{1}{\bar{\gamma}_{\bar{i}}}\right) \gamma_{\mathrm{th}}\right)\right)\right] .
\end{aligned}
$$

Using the transition probabilities, the limiting probabilities can be evaluated and based on them the PDF and CDF expressions (for the high SNR regime) can be directly evaluated using (13) and (14) in (8) and (7), respectively.

\section{SinR Aware Mode Selection: Performance ANALYSIS AND NUMERICAL RESUlTS}

Using the previously derived expressions for the PDF and the CDF of the output SINR (or SIR), the performance of the proposed MS scheme is studied in terms of OP, BEP, and average channel capacity, while a complexity analysis is also performed.

\section{A. Outage Probability $(O P)$}

OP is defined as the probability that the output SINR (or SIR) falls below a predetermined threshold $\gamma_{\mathrm{T}}$ and is given by $P_{\text {out }}=F_{\gamma_{\text {out }}}\left(\gamma_{\mathrm{T}}\right)$. Therefore, using all the CDF expressions that have been derived in the previous section, i.e., (5), (11), and (14), in (7), the OP can directly be evaluated for the SINR, SIR, and high SNR cases, respectively.

\section{B. Average Bit Error Probability (BEP)}

The BEP will be evaluated for the two special cases studied in the previous section, using the PDF-based approach. In general, the BEP can be evaluated as [29]

$$
\bar{P}_{\text {be }}=\int_{0}^{\infty} P_{e}(\gamma) f_{\gamma_{\text {out }}}(\gamma) d \gamma,
$$

where $P_{e}(\gamma)$ denotes the conditional error probability of the modulation scheme. Here, for analytical tractability purposes, we focus on differentially binary phase shift keying (DBPSK), whose conditional error probability is $P_{e}(\gamma)=\exp (-\gamma) / 2$.
1) SIR Use Case: The BEP of DBPSK can be expressed in closed form as follows

$$
\begin{aligned}
& \bar{P}_{\mathrm{be}}=\frac{1}{2}\left\{\sum _ { i = 1 } ^ { 2 } \mathcal { S } _ { 1 } \mathcal { S } _ { 2 } \overline { \gamma } _ { \overline { i } } \overline { \gamma } _ { I _ { j _ { 2 } } , \overline { i } } \sum _ { p = 1 } ^ { 2 } \sum _ { n = 1 } ^ { b _ { i } } \Xi _ { i , p , n } \left[\exp \left(a_{i_{p}}\right)\right.\right. \\
& \times\left[\Gamma\left(2-n, a_{i_{p}}\right)-\Gamma\left(2-n, a_{i_{p}}+\gamma_{\mathrm{th}}\right)-a_{i_{p}} \Gamma\left(1-n, a_{i_{p}}\right)\right. \\
& \left.+a_{i_{p}} \Gamma\left(1-n, \gamma_{\mathrm{th}}+a_{i_{p}}\right)\right]+\sum_{i=1}^{2} \pi_{i}\left[\sum_{p=1}^{2} \mathcal{S}_{3-m_{d}} F_{\gamma_{i}}\left(\gamma_{\mathrm{th}}\right)^{p-1}\right. \\
& \left.\left.\times \bar{\gamma}_{m_{d}}\left[\frac{\exp \left(-\gamma_{\mathrm{th}}\right)}{\gamma_{\mathrm{th}}+a_{i_{p}}}+\exp \left(a_{i_{p}}\right) \operatorname{Ei}\left(-\gamma_{\mathrm{th}}-a_{i_{p}}\right)\right]\right]\right\},
\end{aligned}
$$

where $m_{d}=\bmod (i p, 3), b_{1}=2, b_{2}=1, \Xi_{i, p, n}$ is defined in (A-4). The proof of (17) is provided in Appendix B.

2) High SNR scenario: Substituting (8), (13), (14), and $P_{e}(\gamma)$ in (16), and after some mathematical manipulations, the following asymptotic closed-form expression for the BEP is deduced

$$
\begin{aligned}
& \bar{P}_{\mathrm{be}} \cong \frac{1}{2}\left[\sum_{i=1}^{2} \frac{F_{\gamma_{i}}\left(\left(1+\bar{\gamma}_{i}\right) \gamma_{\mathrm{th}}\right)}{1+\bar{\gamma}_{i}}\right. \\
& \left.-\frac{1-\exp \left[-\left(1+\frac{1}{\bar{\gamma}_{i}}+\frac{1}{\bar{\gamma}_{\bar{i}}}\right) \gamma_{\mathrm{th}}\right]}{\bar{\gamma}_{i}+\bar{\gamma}_{\bar{i}}+\bar{\gamma}_{i} \bar{\gamma}_{\bar{i}}}\right] \\
& \times\left(\bar{\gamma}_{i}+\bar{\gamma}_{\bar{i}}\right)\left[\prod_{i=1}^{2} \mathcal{S}_{i}\left(\bar{\gamma}_{I_{j_{1}, i}}+\bar{\gamma}_{I_{j_{1}, i}}^{2}\right)\right]+\frac{1}{2} \sum_{i=1}^{2} \mathcal{S}_{i} \\
& \times\left(\bar{\gamma}_{I_{j_{1}, i}}+\bar{\gamma}_{I_{j_{1}, i}}^{2}\right) \frac{\exp \left(-\frac{\left(1+\bar{\gamma}_{i}\right) \gamma_{\mathrm{th}}}{\bar{\gamma}_{i}}\right)}{1+\bar{\gamma}_{i}}\left(\pi_{i}+F_{\gamma_{\bar{i}}}\left(\gamma_{\mathrm{th}}\right) \pi_{\bar{i}}\right) .
\end{aligned}
$$

\section{Ergodic Capacity}

The ergodic channel capacity is defined as

$$
\bar{C}=\int_{0}^{\infty} B W \log _{2}(1+\gamma) f_{\gamma_{\text {out }}}(\gamma) d \gamma,
$$

where $B W$ is signal's transmission bandwidth. For the SIR scenario, based on the approach presented in Appendix C, the following closed-form expression for the capacity is extracted

$$
\begin{gathered}
\bar{C}=\left\{\sum_{i=1}^{2} \mathcal{S}_{1} \mathcal{S}_{2} \bar{\gamma}_{\bar{i}} \bar{\gamma}_{I_{j_{\bar{i}}}, \bar{i}} \sum_{p=1}^{2} \sum_{n=1}^{b_{i}} \Xi_{i, p, n}\left(C_{i, p, n}-D_{i, p, n}\right)\right. \\
\left.+\sum_{i=1}^{2} \pi_{i}\left[\sum_{p=1}^{2} \mathcal{S}_{3-m_{d}} F_{\gamma_{i}}\left(\gamma_{\mathrm{th}}\right)^{p-1} \bar{\gamma}_{m_{d}} \bar{\gamma}_{I_{j_{p}}, 3-m_{d}} \mathcal{G}\right]\right\} B W,
\end{gathered}
$$

where

$$
\begin{aligned}
& \mathcal{G}=\left\{\bar{\gamma}_{m_{d}} \ln \left(\frac{\bar{\gamma}_{I_{j_{p}}, 3-m_{d}}}{\bar{\gamma}_{I_{j_{p}}, 3-m_{d}} \gamma_{\mathrm{th}}+\bar{\gamma}_{m_{d}}}\right)+\left[\left(1+\gamma_{\mathrm{th}}\right) \ln \left(1+\gamma_{\mathrm{th}}\right)\right.\right. \\
& \left.\left.+\gamma_{\mathrm{th}} \ln \left(\frac{\bar{\gamma}_{I_{j_{p}}, 3-m_{d}}}{\bar{\gamma}_{I_{j_{p}}, 3-m_{d}} \gamma_{\mathrm{th}}+\bar{\gamma}_{m_{d}}}\right)\right] \bar{\gamma}_{I_{j_{p}}, 3-m_{d}}\right\} \\
& \times\left[\ln (2)\left(\bar{\gamma}_{I_{j_{p}}, 3-m_{d}}-\bar{\gamma}_{m_{d}}\right)\left(\bar{\gamma}_{m_{d}}+\gamma_{\mathrm{th}} \bar{\gamma}_{I_{j_{p}}, 3-m_{d}}\right)\right]^{-1},
\end{aligned}
$$




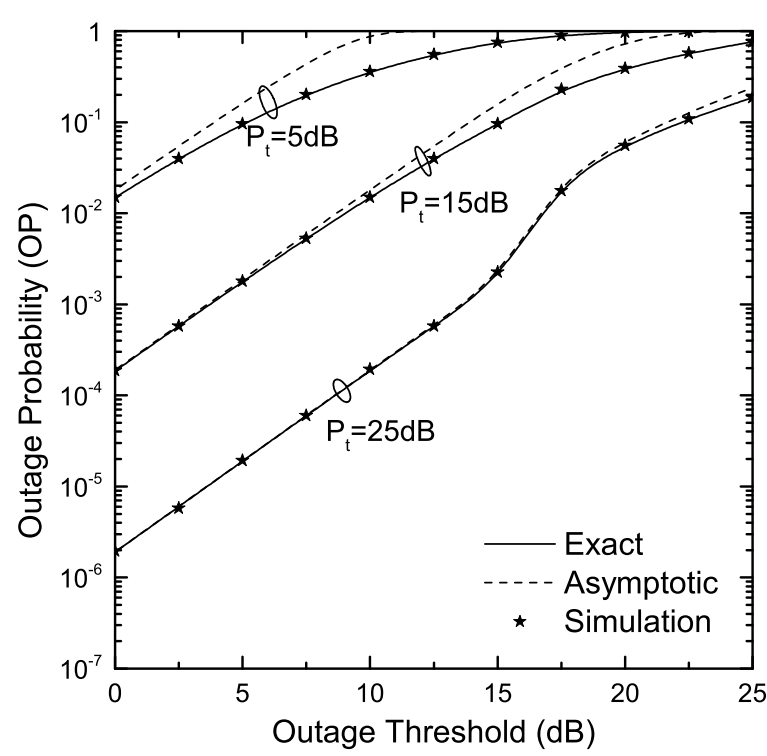

Fig. 2: OP vs $\gamma_{T}$, for different values of $P_{t}$.

and $C_{i, p, 1}=\frac{\ln \left(\gamma_{\mathrm{th}}+1\right)\left(\gamma_{\mathrm{th}}+1\right)-\gamma_{\mathrm{th}}}{\ln (2)}, C_{i, p, 2}=\frac{\operatorname{Li}_{2}\left(\frac{\gamma_{\mathrm{th}}+1}{1-a_{i_{p}}}\right)}{\ln (2)}$ $\frac{\ln \left(\gamma_{\mathrm{th}}+1\right) \ln \left(\frac{a_{i_{p}}+\gamma_{\mathrm{th}}}{a_{i_{p}}}\right)-\mathrm{Li}_{2}\left(\frac{1}{1-a_{i_{p}}}\right)}{\ln (2)}, \quad D_{i, p, 1}=a_{i_{p}} C_{i, p, 2}$, $D_{i, p, 2}=\frac{a_{i_{p}}}{2}\left(\frac{\ln \left(\gamma_{\mathrm{th}}\right)+\ln \left(a_{i_{p}}\right)-\ln \left(a_{i_{p}}+\gamma_{\mathrm{th}}\right)}{a_{i_{p}}-1}-\frac{\ln \left(\gamma_{\mathrm{th}}+1\right)}{a_{i_{p}}+\gamma_{\mathrm{th}}}\right)$, with $\operatorname{Li}_{2}(\cdot)$ denoting the dilogarithm function [30, eq. (27.7.1)].

\section{Complexity Analysis}

The complexity of the proposed scheme is linear related with the average number of path estimations. More specifically, the required number of path estimations $N$ of the proposed scheme can be evaluated as

$$
N=\pi_{1}\left(1+F_{\gamma_{1}}\left(\gamma_{\mathrm{th}}\right)\right)+\pi_{2}\left(1+F_{\gamma_{2}}\left(\gamma_{\mathrm{th}}\right)\right) .
$$

Moreover, it should be noted that an increase on $\gamma_{\text {th }}$ will also result to an increase of the SOs, and thus an increase on the number of signals exchanges. In order to clearly understand the mechanism of SOs, the switching probability (SP) is also analytically evaluated as $P_{s}=\pi_{1}\left(1-P_{11}\right)+\pi_{2}\left(1-P_{22}\right)$.

\section{E. Numerical and Simulation Results}

In this section, using the derived performance analytical results presented in Section IV-A,B,C, several numerical investigations are obtained and presented. In the following, we assume a 2D network topology, where the location of all nodes is determined by their coordinates in a 2D space, [31], with distances normalized to unity. In particular, for obtaining Figs. 2-4, different scenarios regarding the locations of the D2D Txs and Rxs as well as eNB have been assumed, based on the channel model presented in Section II with $\alpha=3$. Such an approach facilitates i) the understanding of the network topology and ii) the focusing on the impact of other (than the distance) parameters of interest. Fig. 2 depicts the OP versus the outage threshold $\left(\gamma_{T}\right)$, using (5) and (7), for the general scenario where both noise and interfering effects are

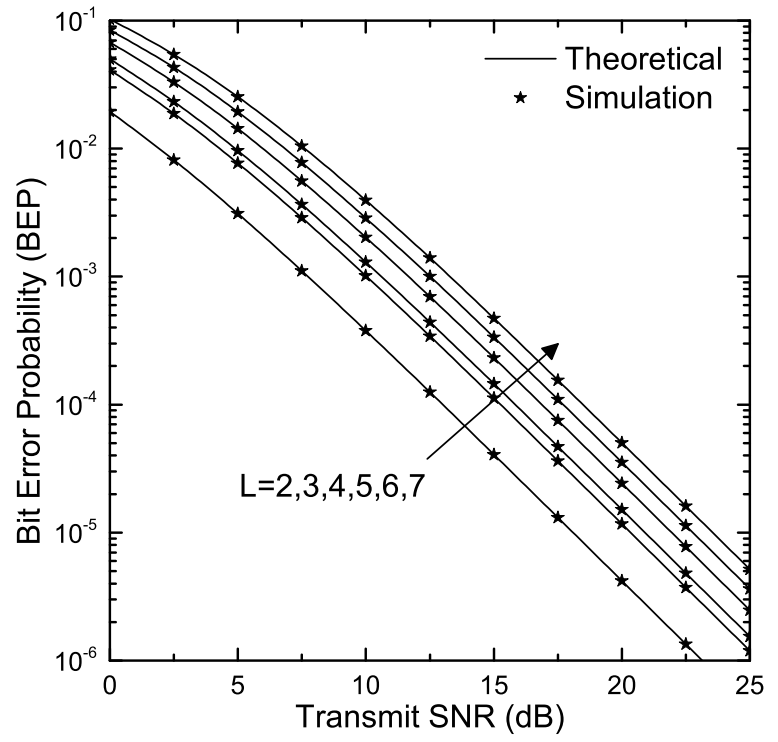

Fig. 3: Average BEP vs $P_{t}$, for different values of $L$.

present. For obtaining this figure, the following locations for the network elements have been considered: D2D Tx $(0,0)$, D2D Rx $(0,0.33)$, eNB $(0,1)$, interferer $I_{1}(1,0.25), I_{2}(1,0.5)$, $I_{3}(1,0.9)$, and $I_{4}(1,1)$, while $P_{I}=0 \mathrm{~dB}, \gamma_{\text {th }}=15 \mathrm{~dB}$. It is shown that the performance improves with an increase on $P_{t}$. Moreover, for comparison purposes, the corresponding asymptotic expression, obtained using (14), is also plotted. It is shown the closed-agreement between the exact and the asymptotic expressions, even for moderate values of $\bar{\gamma}_{i}$.

Fig. 3 provides results for the SIR scenario and illustrates the BEP as a function of the transmit SNR $\left(P_{t}\right)$ for different values of the number of interferers $L$. For obtaining this figure, (17) has been employed, switching threshold is set to $\gamma_{\text {th }}=$ $10 \mathrm{~dB}$, while the following locations have been considered for the D2D Tx $(0,0)$, D2D Rx $(0,0.4)$, and eNB $(0,1)$. As far as the interferers' locations are concerned, six different scenarios have been assumed:

- $I_{1}(1,0.2), I_{2}(1,0.6)$;

- $I_{1}(1,0.33), I_{2}(1,0.5) I_{3}(1,0.6)$;

- $I_{1}(1,0), I_{2}(1,0.33), I_{3}(1,0.66), I_{4}(1,1)$;

- $I_{1}(1,0), I_{2}(1,0.25), I_{3}(1,0.5), I_{4}(1,0.75), I_{5}(1,1)$;

- $I_{1}(1,0), I_{2}(1,0.15), I_{3}(1,0.3), I_{4}(1,0.6), I_{5}(1,0.75), I_{6}$ $(1,1)$

- $I_{1}(1,0), I_{2}(1,0.15), I_{3}(1,0.3), I_{4}(1,0.55), I_{5}(1,0.6), I_{6}$ $(1,0.75), I_{7}(1,1)$.

In this figure, it is shown that the performance decreases with the increase of the number of interferers. It is interesting to note that the difference between the performances is greater for lower values of $L$.

Fig. 4 presents results also for the SIR scenario. More specifically, using (20), the capacity is plotted versus $P_{t}$ for different values of the switching threshold $\gamma_{\mathrm{th}}$. Moreover, for comparison purposes, the corresponding performance of an alternative scheme where in $T_{r r m}$ period the mode of operation offering the best SIR is selected. It is obvious that such a scheme offers the best performance with the cost of increased signal exchange operations. In addition, 


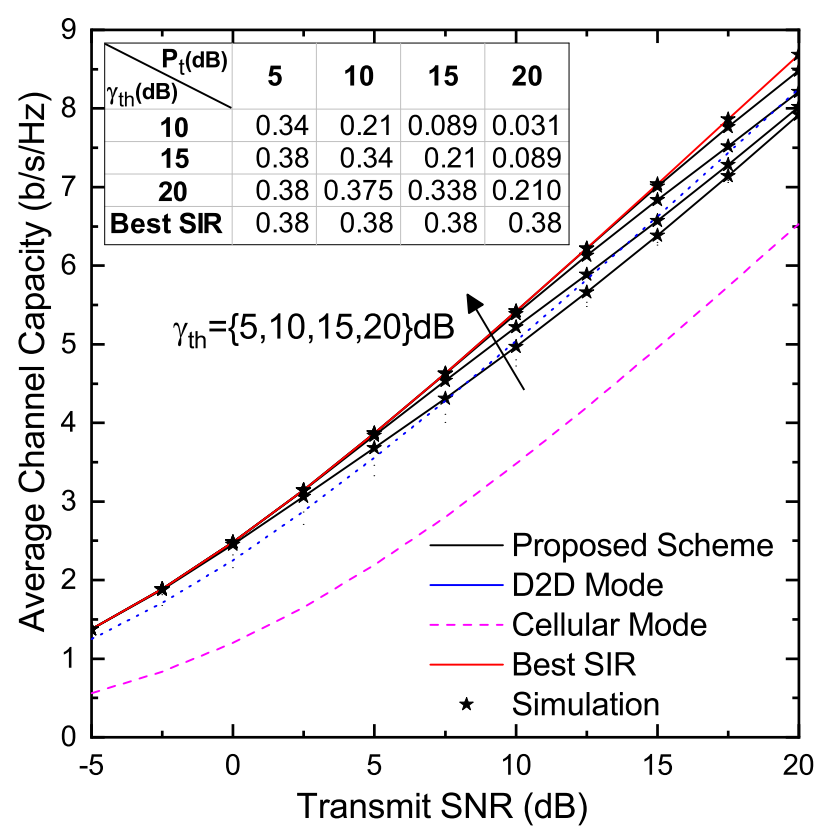

Fig. 4: Average capacity versus transmit power $P_{t}$, for different values of $\gamma_{\mathrm{th}}$.

the performances of single cellular and D2D modes are also plotted. For obtaining this figure, the following locations for the network elements have been considered: D2D Tx $(0,0)$, D2D Rx $(0,0.4)$, eNB $(0,1), I_{1}(1,0), I_{2}(1,0.33), I_{3}(1,0.66)$, $I_{4}(1,1)$. In this figure, it is shown that the performance of the proposed scheme improves with an increase on $\gamma_{\mathrm{th}}$, approaching the corresponding one of the best SIR. Moreover, the performance of D2D mode is always better as compared to that of the cellular mode due to the proximity to the UE. In the table included in Fig. 4, the SP is evaluated for different values of $P_{t}$ and $\gamma_{\mathrm{th}}$ and for the best SIR scheme. From this table, it is shown that SP increases with an increase of $\gamma_{\text {th }}$, approaching that of the best SIR. Therefore, the increase of $\gamma_{\text {th }}$ will result to an improved performance, with the cost of a higher number of SOs. The latter is responsible for an increase of the overhead and/or signal processing, which are required for performing the SOs [32]. By comparing the results depicted in both the figure and the table, it can be concluded that the proposed MS scheme offers an excellent compromise between performance and complexity as compared to best SIR, since for $\gamma_{\mathrm{th}}=P_{t}=10 \mathrm{~dB}$ with a small deterioration on the performance i.e., 3.7\%, a $45 \%$ reduction on the SP is achieved. Finally, Monte Carlo simulation results are also included in all figures, verifying the validity of the proposed theoretical approach.

\section{Multi-User Mode Selection, Scheduling, And RESOURCE AlLOCATION}

In this section, we propose a joint MS, RA, and scheduling solution for the UL using the previously described SINRaware MS approach. The joint investigation of MS and RA is necessary in real-world MU communication scenarios. This is proved by the fact that if MS is performed by each DUE independently, using the SINR threshold rule, then there is a non- negligible probability that multiple D2D pairs may attempt to simultaneously use a sub-channel with low interference. As a consequence, the interference levels will be significantly increased and the system will fail to perform as expected. Thus, MS should be addressed jointly with RA and scheduling, in order to ensure that performance gains for all UEs will be achieved, while compelling an overall system performance improvement. It is noted that the proposed joint MS, RA, and scheduling scheme considers limited SINR-awareness. Therefore, it is not assumed that complete SINR knowledge is available at the scheduler, which may result to errors and inaccuracies.

RA and scheduling in the DL can be performed with any of the conventional methods. However, the investigated use case considers an uplink-limited system. This assumption is generally reasonable since DUEs are systems with low transmit power and limited receiver sensitivity. Moreover, since D2D mode coexists with UL, UL resources are expected to be more congested. Another significant benefit of direct D2D communications is that DL de-congests significantly. In case of a DL-limited system (e.g., heavy internet DL traffic from CUEs), the UL scheduler can be also used to reduce DL traffic. The eNB will prohibit the use of the cellular mode for DUEs by setting UL SINR to $\rightarrow-\infty(\mathrm{dB})$, enforcing them to use the D2D mode.

\section{A. MU System Model Extensions}

In this section, the system model is extended in order to propose the joint MS, RA, and scheduling algorithm. The scheme is designed with no resource sharing between cellular and D2D modes, while reuse of resources is permitted in D2D mode. The following design features are considered:

- MS, RA, and scheduling procedures are performed by the cellular eNB;

- The number of subchannels assigned to cellular and D2D operation mode dynamically changes based on the network conditions, i.e., the eNB is allowed to allocate a variable number of resources to each mode;

- Limited SINR-awareness is assumed. A signaling scheme is used for the exchange of SINR information of the D2D SLs. The assumed procedure is similar with the channel quality indicator (CQI) mechanism in LTE Proximity Services (ProSe), using the physical SL shared or control channels (PSCCH, PSSCH) [25]. The signaling overhead is taken into account in the MS procedure.

The eNB allocates a set of $N_{K}$ subchannels, $K=$ $\left\{K_{n} \mid n=1,2, \ldots, N_{K}\right\}$ organized into a time-frequency grid with $T_{r r m}$ period. These resources are allocated among all users, in cellular or D2D modes. Thus, at a given time, $N_{K_{C}}(t)$ are used for UL transmission while $N_{K_{D}}(t)$ resources are used by D2D SLs, i.e., $N_{K_{C}}(t)+N_{K_{D}}(t)=N_{K}$. As indicated by the time dependence of $N_{K_{C}}(t)$ and $N_{K_{D}}(t)$, the eNB is able to change the number of resources used for each mode of operation. The eNB may allocate up to $M_{C}$ subchannels per UE in cellular mode and $M_{D}$ subchannels per UE in D2D mode for the $T_{r r m}$ period. In addition, it is assumed that a subset of the allocated resources per user is used for signaling. 
For each interval $T_{r r m}$, a signaling overhead (in bps) $R_{\mathrm{sig}, C}$ and $R_{\mathrm{sig}, D}$ is required for cellular and $\mathrm{D} 2 \mathrm{D}$ users, respectively.

The type and number of UEs are also time varying, i.e., $L_{C}(t), L_{D}(t), \mathcal{D}(t), \mathcal{C}(t), \mathcal{G}(t)$ etc. In cellular mode, the eNB is always the second peer of a link, and thus it is able to measure the UL SINR for all UEs of $\mathcal{G}(t)$ operating. Moreover, the eNB is able to measure the UL SINR for UEs operating in D2D mode through the signaling feedback channels. It is also assumed that the eNB cannot measure frequency selective SINR, since the UEs operate only on specifically allocated subchannels. Thus, the eNB uses for MS, RA, and scheduling a single SINR value $\gamma_{G_{i}}(t)$ (wideband SINR) per user, where $G_{i}$ is a user operating in cellular mode $\left(G_{i} \in \mathcal{G}\right)$. The extension of the model for frequency-selective RA is straight forward assuming different SINR values per subchannel.

In this study, it is assumed that the D2D resources are granted by the eNB every $T_{r r m}$ using shared or control channels defined by the protocol. As an example, in LTE ProSe, $T_{r r m}$ can be defined as an integer multiple of the LTE slot and the allocation information can be exchanged through PSCCH, PSSCH. In Fig. 5, a block diagram is presented, illustrating the set of actions performed by the eNB and the UEs in order to enable the joint MS, RA, and scheduling concept. More specifically, the eNB performs MS and assigns the available subchannels using UL SINR measurements and D2D SINR information collected by the devices. The joint MS, RA, and scheduling algorithm performs interference management in order to achieve a configuration that optimizes system performance.

Each DUE's Rx measures the Rx power from other adjacent established D2D links. Thus, for each Rx DUE, a Rx power vector $\mathbf{p}_{D}(t)$ with $L_{D}(t)$ elements is defined

$$
\mathbf{p}_{D_{i}}(t)=\left[p_{D_{1}}(t), \ldots, p_{D_{L_{D}}}(t)\right]^{T}
$$

where the element $p_{D_{j}}$, with $j=1, \ldots, L_{D}(t)$, contains the Rx power from the Tx $D_{j}^{T x}$ of the $j$-th D2D pair. If the received power from a specific DUE Tx, e.g., $D_{j_{0}}^{T x}$, cannot be measured, then it is assumed that $p_{D_{j_{0}}}=N_{0}$. This practically means that the DUE $D_{j_{0}}^{T x}$ is out of coverage. According to Fig. 5, this information is sent to the eNB as feedback.

The eNB retains a MS vector for all managed $L_{D}(m)$ devices, defined as

$$
\begin{aligned}
& \mathbf{v}(m)=\left[\begin{array}{lll}
v_{1}(m), & \ldots & , v_{L_{D}(m)}(m)
\end{array}\right]^{T} \\
& v_{j}(m)=\left\{\begin{array}{c}
1, \text { if } D_{j} \in \mathcal{D}_{1}(\text { DUE in Mode 1) } \\
2, \text { if } D_{j}^{(T x)} \in \mathcal{G} \text { (DUE in Mode 2). }
\end{array}\right.
\end{aligned}
$$

Vector $\mathbf{v}(m)$ retains the current mode status for all D2D pairs. In compliance with LTE ProSe, the eNB is allowed to allocate multiple subchannels per D2D pair. $M_{D}$ is the maximum number of subchannels that can be granted per D2D pair for the $T_{r r m}$ time interval.

\section{B. SINR-Aware MS, RA, and Scheduling Scheme}

In this subsection, the joint MS, RA, and scheduling scheme is analyzed. Since MS and RA is performed in $T_{r r m}$ intervals,

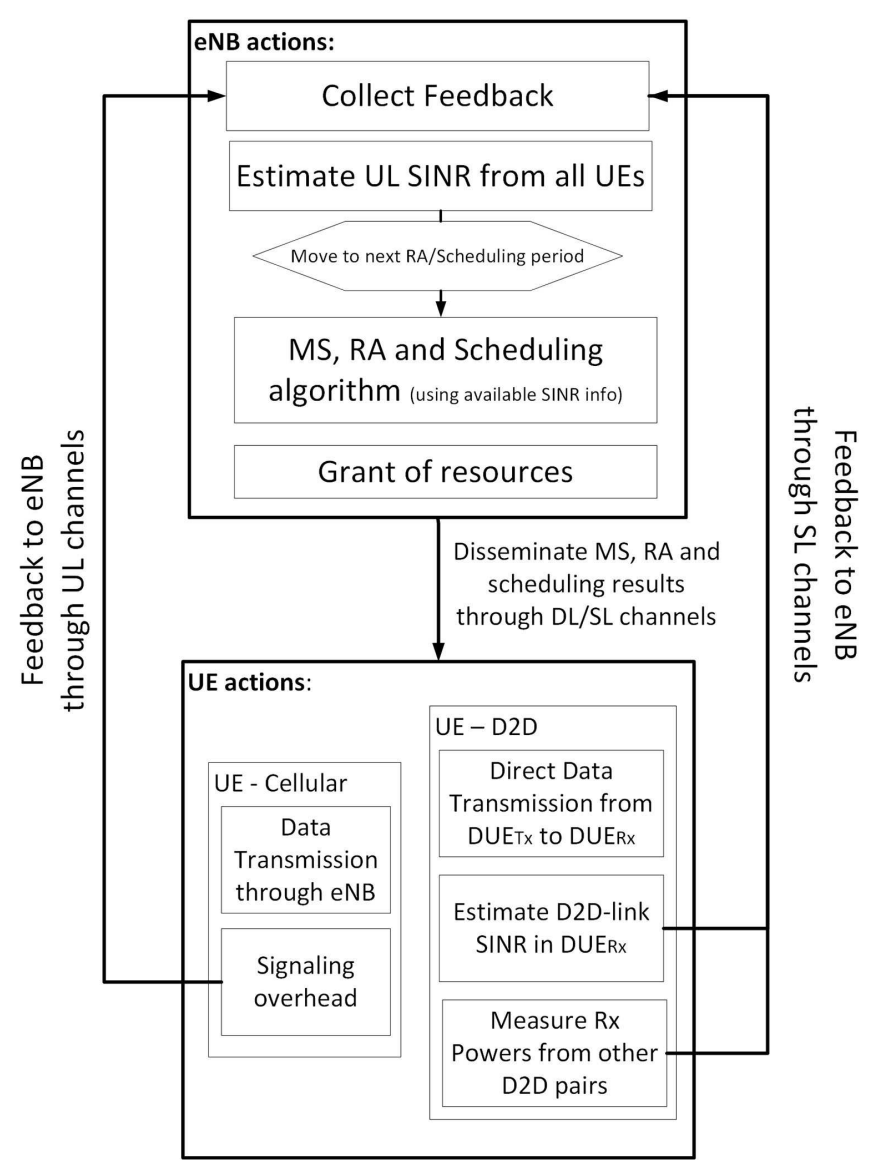

Fig. 5: Block diagram of the joint MS, RA, and scheduling scheme.

the time axis is discretized accordingly, i.e., MS, RA, and scheduling is performed every $m T_{r r m}$ with $m \in \mathbb{Z}$. For brevity, the time interval $T_{r r m}$ will be omitted from the notation in the following relationships. The MS, RA, and scheduling scheme is the procedure that determines the values of a parameter set, that best fits to the objectives of the scheme, i.e., that maximizes a defined utility function. Based on the specified system model, the parameter set $\mathbf{p}$ includes the following variables: i) the number of subchannels allocated in the cellular mode $N_{K_{C}}(m)$, ii) the number of subchannels allocated in D2D mode $N_{K_{D}}(m)$, iii) the selected mode $\mathbf{v}(m)$ for all D2D pairs, iv) the set of allocated subchannels per CUE $\mathcal{I}_{C_{i}}$, v) the set of cellular mode subchannels allocated to DUEs $\mathcal{I}_{D_{i, 2}}$, and vi) the set of D2D subchannels allocated to DUEs $\mathcal{I}_{D_{i, 1}}$.

The eNB manages the joint procedure. However, the eNB has no direct knowledge of the D2D links SINR. Thus, an SINR reporting mechanism from the D2D pairs to the eNB is necessary for supporting the SINR-aware MS procedure. In this study, it is assumed that for each D2D connection, extra signaling content is required. The signaling information contains a list with i) the SINR for the desired instantaneous D2D SL $\gamma_{D_{j}}$ and, ii) the list of the received signal powers from the $\hat{L}$ more powerful D2D neighbors, i.e., the $\hat{L}$ elements of (22) with the higher values. The eNB will use the feedback information to estimate the SINR for the D2D SL subchannels. 
In practical cases, the signaling factor $\hat{L}$, i.e., the number of $\mathrm{D} 2 \mathrm{D}$ interferers, is less than the total number of device pairs operating in the system. Thus, if $\hat{L} \leq L_{D}(m)-1$, the specified methodology defines a joint MS, RA, and scheduling scheme with limited SINR awareness. Moreover, the signaling overhead depends on the parameter $\hat{L}$. The overhead in bps for active D2D pairs is assumed to be $R_{\mathrm{sig}, D}(\hat{L})$.

According to the new scheme, the decision rule that specifies a mode change attempt is provided in the input argument of (3), i.e. when a device pair operates in the $i$-mode, a decision for a mode change attempt to mode $\bar{i}$ is taken when:

$$
\gamma_{i}<\gamma_{\text {th }} \text { and } \gamma_{\overline{\mathrm{i}}} \geq \gamma_{\mathrm{i}}
$$

A device pair that currently operates in cellular mode $(i=2$, i.e., $D_{j}^{T x} \in \mathcal{G}$ ) will be considered for D2D operation, when the SINR of the cellular link falls below the predefined QoS SINR threshold $\gamma_{\text {th }}$ and simultaneously the SINR of the D2D SL is higher. On the other hand, a device pair currently operating in D2D mode $\left(i=1, D_{j} \in \mathcal{D}_{1}\right)$ will request dissolution of the SL and establishment of cellular links, if the SINR of the D2D SL falls below $\gamma_{\text {th }}$ and simultaneously the SINR of the $\mathrm{UL}$ is higher. It is noted that the request for mode change will proceed, even if $\gamma_{\bar{i}}<\gamma_{\mathrm{th}}$. Since the devices will measure and check the SINR for both modes, they will attempt to use the mode with the best SINR, even if it is less than $\gamma_{\text {th }}$.

The eNB can deny the D2D MS request based on the results of the RA and scheduling algorithm. In D2D communications, low latency is a critical aspect. Therefore, a proportional fair (PF) scheduler is assumed, in order to fairly distribute resources among all users. For any link from node $X$ to $Y$ (either UL or SL), the PF function is defined as

$$
f_{X, Y}(m)=\frac{R_{X, Y}(m)-R_{\text {sig }}}{\bar{R}_{X, Y}},
$$

where $R_{X, Y}(m)$ is the instantaneous achievable throughput for the $m$-th RA period, $\bar{R}_{X, Y}$ is the average achieved data rate and $R_{s i g}$ is the signaling overhead for the specific mode. The total instantaneous throughput over all assigned subchannels for a specific link is given by

$$
R_{X, Y}(m)=\sum_{n \in \mathcal{I}_{X Y}} r_{X Y, n}(m),
$$

where $\mathcal{I}_{X Y}$ is the set of allocated subchannels to the link $\mathrm{X}, \mathrm{Y}$ and $r_{X Y, n}$ the instantaneous throughput for subchannel $n$. The channel capacity formula multiplied with $T_{r r m}$ period is then used to express the instantaneous achievable data rate per allocated subchannel $n$

$$
r_{X Y, n}(m)=B W \log _{2}\left(1+\gamma_{X Y, n}(m)\right) T_{r r m} .
$$

Assuming that no frequency-selective RA is possible between UL and D2D SL and that all subchannels occupy the same bandwidth, then $\gamma_{X Y, n}=\gamma_{i}$ for all $n$ and the total achievable rate is given by

$$
R_{X, Y}(m)=M_{X Y}(m) B W \log _{2}\left(1+\gamma_{i}(m)\right) T_{r r m},
$$

where $M_{X Y}(m)=$ length $\left(\mathcal{I}_{X Y}\right)$ is the number of allocated subchannels for the link between $X$ and $Y$ at the $m$-th $T_{r r m}$ period.

\section{Vi. Joint Mode Selection, Resource Allocation, AND SCHEDULING: Optimization ALgORITHM AND RESULTS}

\section{A. Optimization Algorithm}

Since the PF approach is followed, the utility function calculates the sum of PF-functions for all UEs depending on the allocated subchannels and the selected mode of operation. The utility function for a given parameter set $\mathbf{p}$ is expressed as

$$
\begin{aligned}
& g(m, \mathbf{p})=\left\|\mathbf{r}_{C}(m, \mathbf{p})\right\|_{1}+(2-\mathbf{v}(m)) \mathbf{r}_{D}^{1}(m, \mathbf{p}) \\
& +(\mathbf{v}(m)-1) \mathbf{r}_{D}^{2}(m, \mathbf{p}),
\end{aligned}
$$

where $\|\cdot\|_{1}$ is the Manhattan norm [33] and $\mathbf{r}_{C}(m), \mathbf{r}_{D}^{1}(m)$, $\mathbf{r}_{D}^{2}(m)$ are the PF-metric vectors for CUEs, DUEs, if D2D mode is enabled, and DUEs, if cellular mode is enabled, respectively. The PF-metric vectors are defined using the PF formula of (25) combined with the definitions of (27), and (28). Thus, the PF-metric vectors are defined as:

$$
\begin{aligned}
{\left[\mathbf{r}_{C}(m, \mathbf{p})\right]_{i} } & =\frac{R_{C_{i}, e N B}(m)-R_{s i g, C}}{\bar{R}_{C_{i}}}, i=1, \ldots, L_{C}(m) \\
{\left[\mathbf{r}_{D}^{2}(m, \mathbf{p})\right]_{i} } & =\frac{R_{D_{i}^{T x}, e N B}(m)-R_{s i g, C}}{\bar{R}_{D_{i}}}, i=1, \ldots, L_{D}(m) \\
{\left[\mathbf{r}_{D}^{1}(m, \mathbf{p})\right]_{i} } & =\frac{R_{D_{i}^{T x}, D_{i}^{R x}}(m)-R_{s i g, D}}{\bar{R}_{D_{i}}}, i=1, \ldots, L_{D}(m),
\end{aligned}
$$

where $[\cdot]_{i}$ indicates the content of the $i$-th element of the vector and $\bar{R}_{D_{i}}$ is the average rate for D2D pair $i$ using either of the two available modes.

Optimum MS in the PF sense is achieved with the solution of the following constrained maximization problem

$$
\max _{\mathbf{p} \in \text { Parameter Set }}(g(m, \mathbf{p})),
$$

subject to:

$$
\begin{gathered}
C 1: \mathcal{I}_{X} \cap \mathcal{I}_{Y}=\emptyset, \text { for all } \mathrm{X} \neq \mathrm{Y}, \text { if } \mathrm{X}, \mathrm{Y} \in \mathcal{G} \\
C 2: M_{X}=\text { length }\left(\mathcal{I}_{X}\right) \leqslant M_{C}, \text { if } \mathrm{X} \in \mathcal{G} \\
C 3: M_{X}=\text { length }\left(\mathcal{I}_{X}\right) \leqslant M_{D}, \text { if } \mathrm{X} \in \mathcal{D}_{1} \\
C 4: \quad N_{K}=\sum_{X \in \mathcal{G} \mid X \in \mathcal{D}_{1}} M_{X} .
\end{gathered}
$$

The imposed constraints are used to ensure that:

- $C 1$ : No UL resources are shared between users operating in cellular mode;

- $C 2$ : The number of subchannels allocated per UE operating in cellular mode is less than $M_{C}$;

- $C 3$ : The number of subchannels allocated per active $\mathrm{D} 2 \mathrm{D}$ SL is less than $M_{D}$;

- The total number of allocated resources is equal to $N_{K}$. The optimization problem in (31) is an NP-hard combinatorial problem [34]. Moreover, it must be taken into account that based on the assumed signaling feedback strategy, the eNB will attempt to maximize the utility function (applying resource reuse in D2D mode) having incomplete knowledge of interference levels in D2D receivers. In this work, we propose 
the use of an heuristic greedy algorithm for joint MS, RA, and scheduling. The proposed algorithm sequentially investigates the $N_{K}$ available subchannels (see Algorithm 1). Given the fact that the algorithm is greedy, each investigated subchannel is allocated to the UE or set of UEs that maximize the sum of the PF metrics.

First, the algorithm estimates the achievable data rates for the CUEs using (27). Next, MS is performed using the decision rule of (24). The algorithm determines the operating mode of preference for the DUEs and calculates the achievable data rates for the selected mode. The next step is to proceed with the RA and scheduling procedure. The algorithm spans sequentially the available subchannels. For each subchannel, the PF metrics per UE (both CUEs and DUEs) are calculated and sorted in decreasing order. The UE $X$ with the maximum PF metric is selected and if the number of allocated subchannels $M_{X}$ is less than $M_{C}$ or $M_{D}$ (for CUE or DUE, respectively), then the subchannel is assigned to $X$. In the case of cellular mode, i.e., $X \in \mathcal{G}(m)$, the algorithm moves to the next subchannel, since no reuse is allowed.

If $X \in \mathcal{D}_{1}$, i.e., UE will operate in D2D mode, then subchannel reuse is permitted and the algorithm searches for the set of devices that maximizes the sum PF-metric for the given subchannel. First, the subchannel is allocated to the D2D pair with the highest PF-metric and the subchannel reuse algorithm is activated. Let's define $\mathcal{Q}_{n}$ as the set of D2D pairs assigned in the $n$-th subchannel. Then $\mathcal{Q}_{n}$ is initialized with the D2D pair $X$. As a next step, eNB excludes the $\hat{L}$ more powerful interferers of $X$ from the reuse procedure (set $\Lambda$ contains the excluded pairs). The remaining D2D pairs are sorted in decreasing order using the PF-metrics and the D2D pair $Y_{1}$ with the highest value is selected. If $Y_{1}$ has reached the maximum number of allocated subchannels $M_{D}$, the algorithm moves to the second highest PF metric value. Otherwise, the algorithm examines if the subchannel reuse maximizes the sum of PF-metrics for $X$ and $Y_{1}$.

In the sequel, the sum-rate of the devices that will share the channel has to be extracted. Due to the limited SINR awareness, the eNB is not able to calculate the exact SINR at the receivers of $X$ and $Y_{1}$ D2D pairs. Therefore, the worst case scenario is taken into account. Based on the feedback procedure, eNB retains a list of received powers at the Rx of pair $X$ from the $\hat{L}$ most powerful interferers. If $\mathbf{p}_{x}$ is the $(\hat{L} \times 1)$ vector containing the Rx power from the $\hat{L}$ most powerful DUE neighbors of $X$, then the interference level is bounded by the minimum value of $\mathbf{p}_{x}$

$$
\gamma_{X} \geqslant \frac{p_{X}}{N_{0}+\min \left(\mathbf{p}_{x}\right)},
$$

where $p_{X}$ is the received signal power for the D2D pair $X$. The equality in (32) defines the lowest possible SINR in the Rx of pair $X$, if the $n$-th subchannel is shared between $X$ and $Y_{1}$. Using the worst case SINR, the sum of PFmetrics is calculated for $X$ and $Y_{1}$ and if it exceeds the initial PF metric of $X$, then the eNB allocates the subchannel to both pairs, otherwise the reuse subroutine terminates. If not terminated, the subroutine repeats the procedure trying to find a third pair suitable for subchannel reuse. The worst case
TABLE II: Simulation Parameters

\begin{tabular}{|l|l|}
\hline Parameter & Value \\
\hline$T_{r r m}$ & $10 \mathrm{msec}$ \\
\hline Subchannel bandwidth $B W$ & $1.08 \mathrm{MHz}$ \\
\hline Total bandwidth & $20 \mathrm{MHz}$ \\
\hline Number of time slots per $T_{r r m}$ & 20 \\
\hline Number of usable subchannels over $T_{r r m}$ & 360 \\
\hline UE transmitted power & $20 \mathrm{dBm}$ \\
\hline Noise power & $-108 \mathrm{dBm}$ \\
\hline Mean interference power in cellular mode & $-98 \mathrm{dBm}$ \\
\hline Signaling overhead in cellular mode $R_{\text {sig }, C}$ & $100 \mathrm{bps}$ \\
\hline Signaling overhead in D2D mode $R_{\text {sig, }}$ & $R_{\text {sig, }} \hat{L}$ \\
\hline MS threshold $\gamma_{\text {th }}$ & $12 \mathrm{~dB}$ \\
\hline$M_{C}, M_{D}$ & 20 \\
\hline Pathloss exponent $\alpha$ & 3.5 \\
\hline Number of simulation runs & 1000 \\
\hline Number of $T_{r r m}$ periods per run & 400 \\
\hline
\end{tabular}

SINR is recalculated accordingly, i.e., $N_{0}+2 \min \left(\mathbf{p}_{x}\right)$. The resource sharing algorithm will end if no overall PF-metric improvement is achieved.

\section{B. Simulation Setup and Results}

A simulator that implements the described system model and applies the heuristic greedy algorithm was developed. A cell of $2 \mathrm{~km}$ radius is assumed, where the eNB is located in the center of the area. Two main simulation topologies are considered. In Configuration 1, the location of all UEs in the network is generated randomly using the uniform distribution in the area of coverage, with $1 \mathrm{~km}$ maximum distance between Tx and Rx of a DUE pair. In Configuration 2, CUEs are uniformly distributed, while D2D pairs are produced in five clusters. Each cluster is centered in the specific positions of the four interferers of the scenario analyzed in Fig.2, while one additional cluster is considered in the position of the $\mathrm{Rx}$ for the specific scenario. The locations of the D2D Tx and $\mathrm{Rx}$ are extracted uniformly with a radius of $150 \mathrm{~m}$ from the cluster center (it is noted that in Fig.2 distances are normalized to unity). The clustered topology allows us to compare the performance of a UE in the simulated MU environment with the results presented in Sec. IV-E.

The system has similar transmission characteristics with the $20 \mathrm{MHz}$ LTE-A. The subchannels are groups of six adjacent resource blocks in the frequency domain, therefore each subchannel has $1.08 \mathrm{MHz}$ bandwidth. Period $T_{r r m}$ is set to $10 \mathrm{msec}$ (LTE frame duration), thus the joint MS, RA, and scheduling algorithm is applied on a per frame basis. The eNB is able to assign up to 20 subchannels per user (equal to the number of LTE slots in a frame). It is assumed that some bandwidth is reserved from the protocol (e.g., for signaling, guard bands, reference signals etc.) and that 18 subchannels per slot are used for data transmission, providing 360 available subchannels per $T_{r r m}$. The signaling overhead for cellular mode $R_{\text {sig,C }}$ was set to $100 \mathrm{bps}$, which is equivalent to a typical LTE-A configuration where feedback signals from 


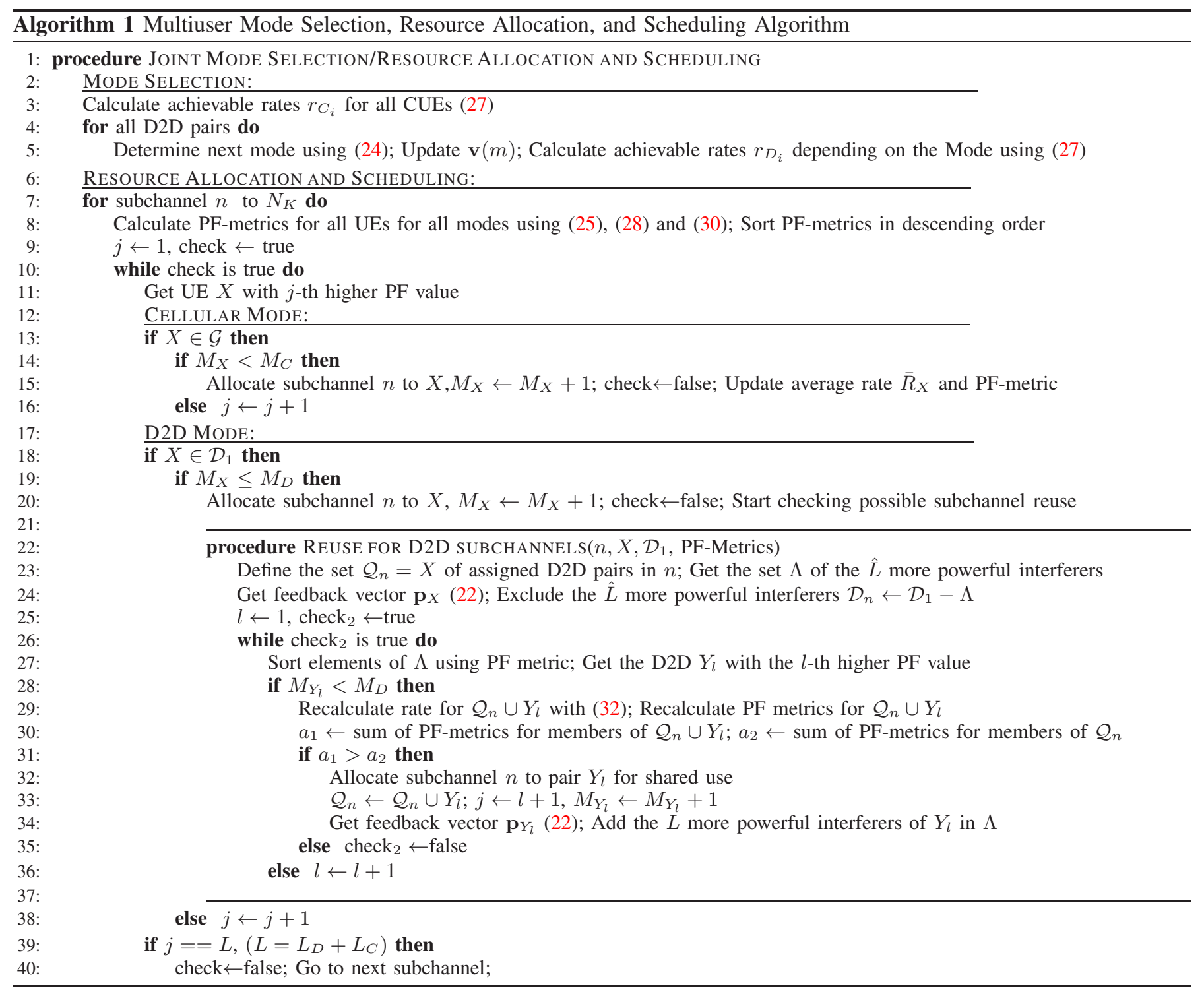

CUEs are multiplexed in the PUCCH (Physical Uplink Control Channel) and reported back to the eNB (in 20msec intervals), [25]. Rayleigh fading conditions for all links are assumed, while the complete parameter set is provided in Table II. The number of CUEs, as well as the number of possible D2D pairs changes dynamically. Two Poisson processes $\lambda_{C, \text { births }}$ and $\lambda_{C, \text { deaths }}$ are defined in order to model births and deaths of CUEs $\left(\lambda_{D, \text { births }}\right.$ and $\lambda_{D \text {,deaths }}$ for the D2D pairs). In order to retain a constant average number of UEs over time, the intensities of the death-birth processes are equal, i.e., $\lambda_{C / D, \text { births }}=\lambda_{C / D, \text { deaths }}$. The number of UEs is updated every $T_{r r m}$.

In Fig. 6, the system sum-rate (in Mbps) vs. average number of D2D pairs is depicted for various signaling overhead factors values $\hat{L}$, while the average number of UEs is $E\left(L_{C}\right)+$ $E\left(L_{D}\right)=150$. As the number of D2D pairs increases, the ability of UEs to operate in D2D mode provides significant improvement in the overall throughput for $\hat{L}=5$ and mainly for $\hat{L}=10$. For $\hat{L}=1$, the eNB is practically unaware of the SINR conditions in D2D mode and favors the use of cellular mode. As a result, the system performance does not change significantly compared with the use of cellular mode only $\left(L_{D}=0\right)$. It should also be noted that for low $L_{D}$ values, the performance for $\hat{L}=5$ is slightly better than $\hat{L}=10$. This is due to the fact that the algorithm will exclude the $\hat{L}$ most powerful devices, which means that for $L_{D}<10$ no reuse in D2D operation is supported with $\hat{L}=10$ and no gain in the system capacity exists. As $L_{D}$ increases, the configuration with $\hat{L}=10$ prevails. Simulation results are presented for both configurations. It can be seen that the results for Configuration 2 is generally better, due to the fact that the specific topology generates low SINR values significantly less frequently.

Furthermore, in Fig. 6, a comparison of the proposed scheme with the optimal scheduler for $\hat{L}=10$ is performed. Every scheduling period, the optimal scheduler assigns the resources based on the allocation that maximizes (29). It is seen that the optimal scheduler presents slightly increased achievable sum-rate compared to the proposed greedy-heuristic algorithm. Nevertheless, the proposed scheme achieves exceptional performance, since the observed difference does not 
exceed $5 \%$ of the achieved sum-rate for all $N_{D}$. It should be noted that the implementation of an optimal scheduler in a practical system becomes impossible due to significant problems and weaknesses. First of all, in order to find the optimal solution, an exhaustive search between every possible allocation and combination of CUEs and DUEs has to be performed. Application of brute-force search in a multi-mode scheduling scenario with $N_{D}+N_{C}$ UEs is an extreme, timeconsuming procedure that involves sequential check of multibillion combinations of possible allocations. More specifically, if $U_{D}$ is the maximum number of DUEs sharing a resource block allocated for D2D operation, and $Q$ is the complexity introduced for the calculation of the SINR, rate and PF-metric, then the number of calculations for the optimal scheduler is given by $Q \frac{N_{D} !}{\left(N_{D}-U_{D}\right) ! U_{D} !}$. For $N_{D}=100$ and $U_{D}=20$, the number is exceptionally high $\left(>10^{13}\right)$. This number can be reduced by excluding the $\hat{L}$ stronger interferers for each DUE allocated in the resource block. Nevertheless, even with this reduction, multi-million calculations are generally required for each resource block. Thus, such a scheduler cannot be applied in a real-world system.

On the other hand, the proposed MS, RA, and scheduling scheme is based on simple calculation and classification of the PF-functions per UE, which is triggered only when the SINR drops below $\bar{\gamma}_{\text {th }}$. Each time the scheduling algorithm is used, the following calculations are made: calculation and sorting of the SINRs, rates and PF-metrics for all UEs; if D2D mode is selected in the resource block, then DUEs are sequentially tested. The test includes sorting and exclusion of $\hat{L}$ most powerful interfereres and re-calculation of SINR, rate, and PF-metric is performed. Assuming $U_{D}$ DUEs allocated in the resource block, the upper bound of the complexity is $Q \times U_{D}$ (with additional $U_{D}$ sorting procedures per resource block). Therefore, the greedy heuristic algorithm is quite practical in terms of computational complexity.

Regarding MS, the optimal scheduler does not use a threshold, but SINR-based selection is performed for all UEs per $T_{r r m}$. DUEs are required to adapt their transmission/reception features, each time MS is triggered. Therefore, MS is also a factor that increases system complexity. During the simulations, MS procedures where counted, assuming $\gamma_{\text {th }}=12$ and $10 \mathrm{~dB}$. The results are presented in Fig. 7, compared to a simplified scheduler that does not use any threshold. The simulation shows that the proposed scheme performs on average $47 \%$ less MS attempts for $\gamma_{\text {th }}=12 \mathrm{~dB}$ and $65 \%$ for $\gamma_{\mathrm{th}}=10 \mathrm{~dB}$ for Configuration 1. Configuration 2 favors D2D mode, since DUEs are generally at smaller distances, and DUEs of the same cluster do not interfere with each other. This bias towards D2D mode reduces the overall number of MS procedures. In addition, since the distribution of DUEs in clusters does not favor low SINRs, MS reduction exceeds $80 \%$. Thus, the use of the greedy heuristic scheme with threshold leads to significant reduction of the system complexity.

In Fig. 8, the simulation results are used to extract the empirical CDFs of the UE SINR. The signaling overhead factor was set to $\hat{L}=10$. Three CDFs are depicted per configuration: UEs in cellular mode only, equal number of CUEs and DUEs, and UEs in D2D mode only. It becomes clear

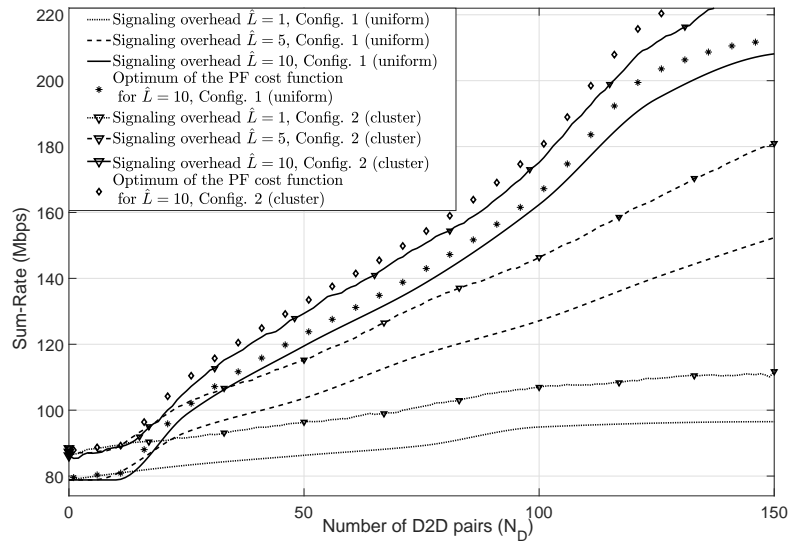

Fig. 6: Sum-rate in Mbps vs $N_{D}$ for various signaling overhead factors $\hat{L}$.

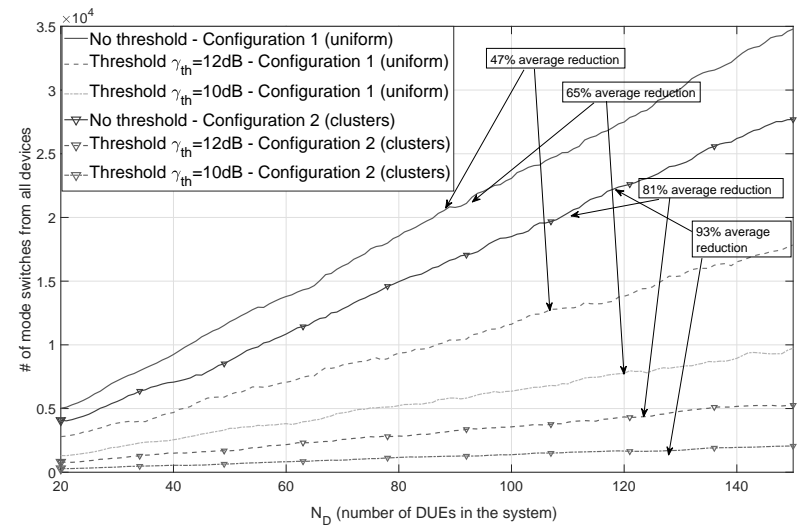

Fig. 7: Number of mode switches vs $N_{D}$ for without using a threshold and for $\gamma_{\text {th }}=10$ and $12 \mathrm{~dB}$ for all configurations.

from Fig. 8, that the average UE SINR increases significantly, as the number of DUEs increases. However, UEs with very low SINR (in both modes of operation) will not benefit from the increase of DUE number. For Configuration 1 and very low SINR, the cellular mode performs slightly better than mode selection. This is a result of limited SINR-awareness, that will lead to erroneous estimation of SINRs for D2D mode. Consequently, the DUE will fail to select the best mode of operation, resulting in performance degradation. Moreover, in very low SINR, estimation and synchronization errors affect system performance, especially in D2D operation, where there is no support from the eNB. On the other hand, in Configuration 2, each DUE is able to successfully exclude all $\hat{L}$ powerful interferers that coexist in its cluster. Therefore, limited SINR-awareness does not have any impact for verylow SNR for this topology.

Fig. 9 depicts the results of OP for a given device in the multiuser environment using both configurations for various $\hat{L}$ values. The average number of D2D pairs $E\left(L_{D}\right)$ is set to 50 and $10 \mathrm{D} 2 \mathrm{D}$ pairs are considered per cluster. It can be seen that for $\hat{L}=10$, the results practically coincide with the performance analysis of SINR-aware MS presented in Fig. 2. Due to the fact that the cluster size is equal to $\hat{L}$, each device 


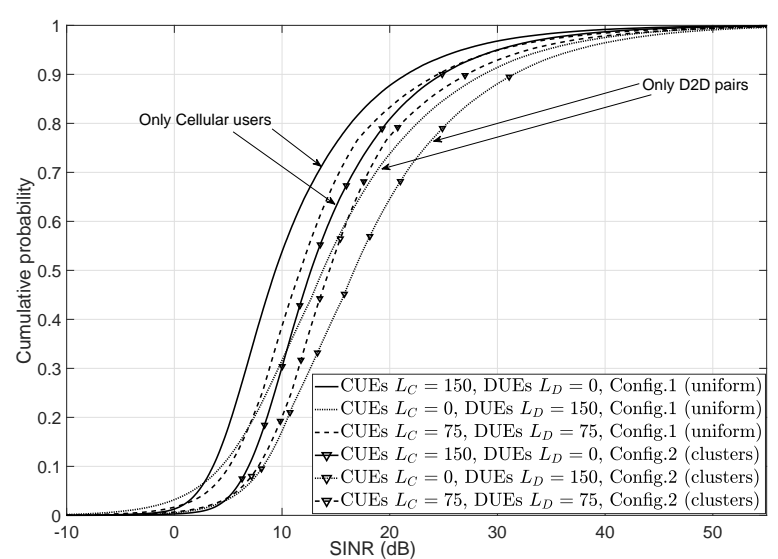

Fig. 8: Empirical CDF for SINR (in dB) for signaling overhead factor $\hat{L}=10$ and various number of CUEs and DUEs.

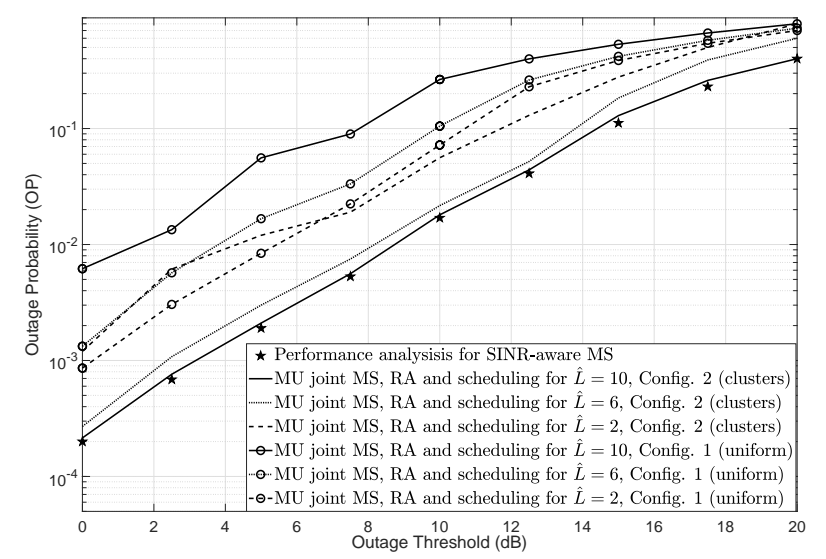

Fig. 9: Outage probability for a given device in multiuser environment for both configurations

typically excludes all the nearest neighbors and the average number of D2D pairs sharing the subchannel is four (one from each cluster). Thus, the performance achieved with the application of the joint MS, RA, and scheduling algorithm with limited SINR-awareness approximates successfully the theoretical performance analysis of SINR-aware MS. On the other hand, for $\hat{L}=2$, the user may coexist with strong interferers and the performance deteriorates significantly. Moreover, the results for Configuration 1 deviate significantly, even for $\hat{L}=10$. However, this is expected since this configuration has different setup with the one in Fig. 2.

\section{CONCLUSIONS}

In this paper, a novel MS technique for D2D enabled communication networks is proposed. The new technique selects the mode of operation that offers an acceptable performance according to the received SINR. In particular, in order to avoid continuously SO, the instantaneous received SINR is compared with a predefined threshold, in order to allow SO only when SINR is below that threshold. The new MS is modeled as a Markov chain and an analytical framework for important statistical metrics, such as the PDF and CDF of the output SINR are presented for the single-user scenario.
Using this analysis, various performance results have been presented, such as the OP, BER, and the channel capacity. Moreover, the new scheme is also applied in a multiuser scenario, where a joint MS, RA, and scheduling optimization problem is formulated. A solution to this problem is provided in terms of a heuristic greedy algorithm. From the derived results it is depicted that the proposed scheme offers clear performance improvement in terms of sum-rate and average UE SINR, with no considerable increase in signaling overhead or complexity.

\section{APPENDIX A \\ DERIVATION OF EQ. (6)}

In this Appendix, a closed-form expression for (6), is provided. This probability can be evaluated as

$$
\begin{aligned}
\operatorname{Pr}\left[\gamma_{i}<\gamma_{\text {th }}, \gamma_{i} \geq \gamma_{j}\right] & =\int_{0}^{\gamma_{\text {th }}} \int_{0}^{x} f_{\gamma_{\bar{i}}}(y) f_{\gamma_{i}}(x) d y d x \\
& =\int_{0}^{\gamma_{\text {th }}} F_{\gamma_{\bar{i}}}(x) f_{\gamma_{i}}(x) d x .
\end{aligned}
$$

Substituting (2) and (5) in (A-1), the following type of integrals appear

$$
\begin{aligned}
& \mathcal{I}_{1}=\int_{0}^{\gamma_{\text {th }}}\left[\frac{1}{\frac{x}{\bar{\gamma}_{i}}+\frac{1}{\bar{\gamma}_{I_{j_{i}}, \bar{i}}}}+\frac{1}{\left(\frac{x}{\bar{\gamma}_{i}}+\frac{1}{\bar{\gamma}_{I_{j_{i}}, \bar{i}}}\right)^{2}}\right] \exp \left(-\frac{x}{\bar{\gamma}_{i}}\right) d x \\
&=\underbrace{\int_{0}^{\gamma_{\text {th }}} \frac{\exp \left(-\frac{x}{\bar{\gamma}_{i}}\right)}{\frac{x}{\bar{\gamma}_{i}}+\frac{1}{\bar{\gamma}_{I_{j_{i}}, \bar{i}}}} d x}_{\mathcal{I}_{1, a}}+\underbrace{\int_{0}^{\gamma_{\mathrm{th}}} \frac{\exp \left(-\frac{x}{\bar{\gamma}_{i}}\right)}{\left(\frac{x}{\bar{\gamma}_{i}}+\frac{1}{\bar{\gamma}_{I_{j_{i}}, \bar{i}}}\right)^{2}} d x}_{0} \\
& \mathcal{I}_{2}=\int_{0}^{\gamma_{\mathrm{th}}\left[\left(\frac{x}{\bar{\gamma}_{i}}+\frac{1}{\bar{\gamma}_{I_{j_{i}}, \bar{i}}}\right)^{-1}+\left(\frac{x}{\bar{\gamma}_{i}}+\frac{1}{\bar{\gamma}_{I_{j_{i}}, \bar{i}}}\right)^{-2}\right]} \\
& \times \exp \left(-\frac{x}{\bar{\gamma}_{i}}\right) \frac{\exp \left(-\frac{x}{\bar{\gamma}_{\bar{i}}}\right)}{\overline{\bar{\gamma}}_{\bar{i}}+\bar{\gamma}_{I_{j_{\bar{i}}}, i} x} d x .
\end{aligned}
$$

In (A-2), $\mathcal{I}_{1, a}, \mathcal{I}_{1, b}$ can be evaluated using [28, eqs. (1.3.2/22) and (1.3.2/26)]. Using these solutions and after some simplifications $\mathcal{I}_{1}$ can be expressed as

$$
\mathcal{I}_{1}=\bar{\gamma}_{i} \bar{\gamma}_{I_{j_{i}}, \bar{i}}\left(1-\frac{\exp \left(-\frac{\gamma_{\mathrm{th}}}{\bar{\gamma}_{i}}\right) \bar{\gamma}_{i}^{2}}{\bar{\gamma}_{i}+\gamma_{\mathrm{th}} \bar{\gamma}_{I_{j_{i}}, \bar{i}}}\right) .
$$

For evaluating $\mathcal{I}_{2}$, the partial fraction approach will be employed as follows

$$
\begin{gathered}
\prod_{i=1}^{2} \frac{1}{\left(a_{q_{i}}+s\right)^{b_{i}}}=\sum_{i=1}^{2} \sum_{n=1}^{b_{i}} \frac{\Xi_{q, i, n}}{\left(a_{q_{i}}+s\right)^{n}} \\
\Xi_{q, i, n}=\left.\frac{1}{\left(b_{i}-n\right) !} \frac{d^{b_{i}-n}}{d x^{b_{i}-n}} \prod_{\substack{p=1 \\
p \neq i}}^{2}\left(x+a_{q_{p}}\right)^{-b_{p}}\right|_{x=-a_{q_{i}}} .
\end{gathered}
$$


Based on this approach and using also [28, eqs. (1.3.2/22) and (1.3.2/26)], $\mathcal{I}_{2}$ can be solved as

$$
\mathcal{I}_{2}=\frac{\bar{\gamma}_{i}}{\bar{\gamma}_{I_{\bar{i}}, i}}\left[\mathcal{H}(1)+\bar{\gamma}_{i} \mathcal{H}(2)\right]
$$

Using these solutions and after some mathematical simplifications, (6) is derived and also completes this proof.

\section{APPENDIX B \\ DERIVATION OF EQ. (17)}

In this Appendix, a closed-form expression for (17), is provided. Substituting (8), (10), (11), and $P_{e}(\gamma)$ for DBPSK in (16), and using the partial fraction approach, integrals of the following form appear

$$
\begin{aligned}
\mathcal{I}_{3} & =\int_{0}^{\infty} \frac{\exp (-\gamma)}{\left(\gamma+a_{1_{2}}\right)^{2}} d \gamma \stackrel{(1)}{=} \frac{1}{a_{1_{2}}}+\exp \left(a_{1_{2}}\right) \operatorname{Ei}\left(-a_{1_{2}}\right) \\
\mathcal{I}_{4} & =\int_{0}^{\gamma_{\mathrm{th}}} \frac{\exp (-\gamma)}{\left(\gamma+a_{1_{2}}\right)^{2}} d \gamma \\
& \stackrel{(2)}{=}-\frac{\exp \left(\gamma_{\mathrm{th}}\right)}{\gamma_{\mathrm{th}}+a_{1_{2}}}+\exp \left(-a_{1_{2}}\right) \operatorname{Ei}\left(-\gamma_{\mathrm{th}}-a_{1_{2}}\right) .
\end{aligned}
$$

In (B-1), for evaluating (1), [28, eq. (2.3.4/4)] has been employed, while for evaluating (2), [28, eq. (1.3.2/26)] has been used. Based on the solutions in (B-1) and after some mathematical manipulations, the closed-form expression for the BEP of DBPSK given in (17) is finally derived.

\section{APPENDix C \\ DERIVATION OF EQ. (20)}

In this Appendix, a closed-form expression for (20), is provided. Substituting (8), (10), and (11), in (19), the following types of integrals appear

$$
\begin{aligned}
& \mathcal{I}_{5}=\int_{0}^{\gamma_{\text {th }}} \frac{\gamma}{\gamma+a_{1_{i}}} \frac{\log _{2}(\gamma+1)}{\left(\gamma+a_{2_{2}}\right)^{2}} d \gamma \\
& \mathcal{I}_{6}=\int_{\gamma_{\text {th }}}^{\infty} \frac{\log _{2}(\gamma+1)}{\left(\gamma+a_{1_{1}}\right)^{2}} d \gamma .
\end{aligned}
$$

For solving $\mathcal{I}_{5}$, first the partial fraction approach is used, i.e.,

$$
\prod_{r=1}^{L}\left(\frac{1}{\gamma+\lambda_{r}}\right)^{m_{r}}=\sum_{r=1}^{L} \sum_{j=1}^{m_{r}} \Xi_{r, j}\left(\gamma+\lambda_{r}\right)^{-j},
$$

where

$$
\Xi_{r, j}=\left.\frac{1}{\left(m_{r}-j\right) !} \frac{d^{m_{r}-j}}{d \gamma^{m_{r}-j}} \prod_{\substack{i=1 \\ i \neq r}}^{L}\left(\gamma+\lambda_{i}\right)^{-m_{i}}\right|_{\gamma=-\lambda_{r}}
$$

and then a change of variables is performed, leading to integrals of the following form

$$
\begin{aligned}
\mathcal{I}_{7_{a}} & =\int_{a_{i_{j}}}^{a_{i_{j}}+\gamma_{\mathrm{th}}} \ln \left(1-a_{i_{j}}+\gamma\right) d \gamma, \\
& \stackrel{(1)}{=}\left[\left(1-a_{i_{j}}+\gamma\right) \ln \left(1-a_{i_{j}}+\gamma\right)-\gamma\right]_{a_{i_{j}}}^{a_{i_{j}}+\gamma_{\mathrm{th}}}
\end{aligned}
$$

$$
\begin{aligned}
\mathcal{I}_{7_{b}} & =\int_{a_{i_{j}}}^{a_{i_{j}}+\gamma_{\text {th }}} \frac{\ln \left(1-a_{i_{j}}+\gamma\right)}{\gamma} d \gamma, \\
& \stackrel{(2)}{=}\left[\ln \left(\left|1-a_{i_{j}}\right|\right) \ln (\gamma)-\operatorname{Li}_{2}\left(-\frac{\gamma}{1-a_{i_{j}}}\right)\right]_{a_{i_{j}}}^{a_{i_{j}}+\gamma_{\text {th }}} \\
\mathcal{I}_{7_{c}} & =\int_{a_{i_{j}}}^{a_{i_{j}}+\gamma_{\text {th }}} \frac{\ln \left(1-a_{i_{j}}+\gamma\right)}{\gamma^{2}} d \gamma \\
& \stackrel{(3)}{=}\left[\frac{\ln (|\gamma|)}{1-a_{i_{j}}}-\frac{1}{\gamma} 0 \frac{1}{1-a_{i_{j}}} \ln \left(\gamma+1-a_{i_{j}}\right)\right]_{a_{i_{j}}}^{a_{i_{j}}+\gamma_{\text {th }}} .
\end{aligned}
$$

In (C-3), for evaluating (1), [28, eq. (1.6.5/4)] has been employed, for evaluating (2), [28, eq. (1.6.5/9)] has been used, and for (3), [28, eq. (1.6.5/13)]. Based on the solutions in (C-3) and after some mathematical manipulations, the closed-form expression for the capacity given in (20) is finally derived.

\section{REFERENCES}

[1] X. Lin, J. G. Andrews, A. Ghosh, and R. Ratasuk, "An overview of 3GPP device-to-device proximity services," IEEE Communications Magazine, vol. 52, no. 4, pp. 40-48, Apr. 2014.

[2] D. S. Gurjar and P. K. Upadhyay, "Performance analysis of zeroforcing-based multiple-input multiple-output two-way relaying in overlay device-to-device communications," IET Communications, vol. 10, pp. 699-708(9), April 2016.

[3] J. Liu, H. Nishiyama, N. Kato, and J. Guo, "On the outage probability of device-to-device-communication-enabled multichannel cellular networks: An RSS-threshold-based perspective," IEEE J. Sel. Areas Commun., vol. 34, no. 1, pp. 163-175, Jan. 2016.

[4] J. Liu, N. Kato, J. Ma, and N. Kadowaki, "Device-to-device communication in LTE-advanced networks: A survey," IEEE Communications Surveys Tutorials, vol. 17, no. 4, pp. 1923-1940, Fourthquarter 2015.

[5] D. S. Gurjar and P. K. Upadhyay, "Three-phase overlay D2D communications in traffic-aware two-way cellular systems," in IEEE Region 10 Conference (TENCON), Nov 2016, pp. 2779-2783.

[6] H. Min, W. Seo, J. Lee, S. Park, and D. Hong, "Reliability improvement using receive mode selection in the device-to-device uplink period underlaying cellular networks," IEEE Trans. Wireless Commun., vol. 10, no. 2, pp. 413-418, Feb. 2011.

[7] K. S. Ali, H. ElSawy, and M. S. Alouini, "On mode selection and power control for uplink D2D communication in cellular networks," in IEEE International Conference on Communication Workshop (ICC), Jun. 2015, pp. 620-626.

[8] H. ElSawy, E. Hossain, and M. S. Alouini, "Analytical modeling of mode selection and power control for underlay D2D communication in cellular networks," IEEE Trans. Commun., vol. 62, no. 11, pp. 41474161, Nov 2014.

[9] D. Marshall, S. Durrani, J. Guo, and N. Yang, "Performance comparison of device-to-device mode selection schemes," in IEEE Personal, Indoor, and Mobile Radio Communications (PIMRC), Aug 2015, pp. 15361541.

[10] A. Galanopoulos, K. Kousias, A. Nikolaidou, F. Foukalas, and T. Khattab, "Energy efficient spectrum allocation and mode selection for mission-critical D2D communications," in IEEE Conference on Computer Communications Workshops (INFOCOM), Apr. 2016, pp. 435440.

[11] Y. Xu, "A mode selection scheme for D2D communication in heterogeneous cellular networks," in IEEE Global Communications Conference (GlOBECOM), Dec 2015, pp. 1-6.

[12] M. Peng, Y. Li, T. Q. S. Quek, and C. Wang, "Device-to-device underlaid cellular networks under Rician fading channels," IEEE Trans. Wireless Commun., vol. 13, no. 8, pp. 4247-4259, Aug 2014.

[13] J. Liu, S. Zhang, H. Nishiyama, N. Kato, and J. Guo, "A stochastic geometry analysis of D2D overlaying multi-channel downlink cellular networks," in IEEE Conference on Computer Communications (INFOCOM), 2015, pp. 46-54.

[14] C. H. Yu, K. Doppler, C. B. Ribeiro, and O. Tirkkonen, "Resource sharing optimization for device-to-device communication underlaying cellular networks," IEEE Trans. Wireless Commun., vol. 10, no. 8, pp. 2752-2763, Aug. 2011. 
[15] H. Pang et al., "Joint mode selection and resource allocation using evolutionary algorithm for device-to-device communication underlaying cellular networks," Journal of communications 8.11, pp. 751-757, 2013.

[16] M. Jung, K. Hwang, and S. Choi, "Joint mode selection and power allocation scheme for power-efficient device-to-device (D2D) communication," in IEEE Vehicular Technology Conference (VTC-Spring), May 2012, pp. 1-5.

[17] G. Fodor and N. Reider, "A distributed power control scheme for cellular network assisted D2D communications," in IEEE Global Telecommunications Conference (GLOBECOM), Dec 2011, pp. 1-6.

[18] K. Yang, S. Martin, L. Boukhatem, J. Wu, and X. Bu, "Energy-efficient resource allocation for device-to-device communications overlaying LTE networks," in IEEE Vehicular Technology Conference (VTC-Fall), Sep. 2015, pp. 1-6.

[19] G. Yu, L. Xu, D. Feng, R. Yin, G. Y. Li, and Y. Jiang, "Joint mode selection and resource allocation for device-to-device communications," IEEE Trans. Commun., vol. 62, no. 11, pp. 3814-3824, Nov 2014.

[20] P. Phunchongharn, E. Hossain, and D. I. Kim, "Resource allocation for device-to-device communications underlaying LTE-advanced networks," IEEE Wireless Communications, vol. 20, no. 4, pp. 91-100, Aug. 2013.

[21] M. H. Han, B. G. Kim, and J. W. Lee, "Subchannel and transmission mode scheduling for D2D communication in OFDMA networks," in IEEE Vehicular Technology Conference (VTC-Fall), Sep. 2012, pp. 1-5.

[22] D. Wu, J. Wang, R. Q. Hu, Y. Cai, and L. Zhou, "Energy-efficient resource sharing for mobile device-to-device multimedia communications," IEEE Trans. Veh. Technol., vol. 63, no. 5, pp. 2093-2103, Jun. 2014.

[23] G. Yu, L. Xu, D. Feng, R. Yin, G. Y. Li, and Y. Jiang, "Joint mode selection and resource allocation for device-to-device communications," IEEE Trans. Commun., vol. 62, no. 11, pp. 3814-3824, Nov. 2014.

[24] P. S. Bithas, A. A. Rontogiannis, and G. K. Karagiannidis, "An improved threshold-based channel selection scheme for wireless communication systems," IEEE Trans. Wireless Commun., vol. 15, no. 2, pp. 1531-1546, Feb 2016.

[25] G. T. 36.211, "Evolved universal terrestrial radio access (E-UTRA); physical channels and modulation (release 13)); overall description," Tech. Rep., 2015.

[26] Y. Huang, A. A. Nasir, S. Durrani, and X. Zhou, "Mode selection, resource allocation, and power control for d2d-enabled two-tier cellular network," IEEE Trans. Commun., vol. 64, no. 8, pp. 3534-3547, Aug 2016.

[27] I. S. Gradshteyn and I. M. Ryzhik, Table of Integrals, Series, and Products, 6th ed. New York: Academic Press, 2000.

[28] A. Prudnikov, Y. Brychkov, and O. Marichev, Integrals and Series, Volume 1. Gordon and Breach Science, 1986.

[29] G. K. Karagiannidis, N. C. Sagias, and T. A. Tsiftsis, "Closed-form statistics for the sum of squared Nakagami-m variates and its applications," IEEE Trans. Wireless Commun., vol. 54, no. 8, pp. 1353-1359, Aug 2006.

[30] M. Abramowitz, I. A. Stegun et al., "Handbook of mathematical functions," Applied mathematics series, vol. 55, p. 62, 1966.

[31] L. Fan, X. Lei, T. Duong, R. Hu, and M. Elkashlan, "Multiuser cognitive relay networks: Joint impact of direct and relay communications," IEEE Trans. Commun., vol. 13, no. 9, pp. 5043-5055, Sep. 2014.

[32] P. Mach, Z. Becvar, and T. Vanek, "In-band device-to-device communication in OFDMA cellular networks: A survey and challenges," IEEE Communications Surveys Tutorials, vol. 17, no. 4, pp. 1885-1922, 2015.

[33] J. E. Gentle, Matrix algebra: theory, computations, and applications in statistics. Springer Science and Business Media, 2007.

[34] M. Pinedo, Scheduling: Theory, Algorithms, and Systems. Prentice Hall, Englewood Cliffs, NJ, 1995.

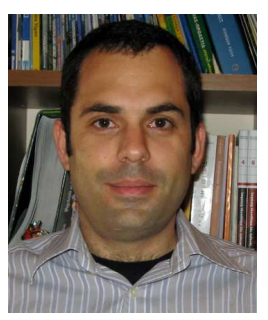

Petros S. Bithas (S'04-M'09) received the B.S. in electrical engineering from the Department of Electrical and Computer Engineering of the University of Patras, Greece, in 2003. From the same Department he received the Ph.D. degree with specialization in "Wireless Communication Systems" in 2009. Since 2010 , he is an associate researcher at the Department of Digital Systems, University of Piraeus, Greece, where he participates in a number of R\&D projects. In May 2018, he joined the Aircraft Engineering Department of the Technological Educational Institute of Sterea Ellada, Greece, as an Assistant Professor. Currently, he is an Assistant Professor at the National and Kapodistrian University of Athens. Professor Bithas serves on the Editorial Board of the International Journal of Electronics and Communications (ELSEVIER). He has been selected as an "Exemplary Reviewer" of the IEEE COMMUNICATIONS LETTERS for year 2010 , while he is also co-recipient of several best paper awards in his areas of research. He has published 34 articles in international scientific journals and 37 articles in the proceedings of international conferences. His current research interests include stochastic modeling of wireless communication channels, as well as design and performance analysis of communication systems.

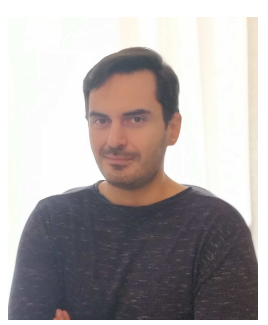

Konstantinos Maliatsos (S'03-M'11) is a senior researcher for the Telecommunications Systems Laboratory of University of Piraeus with experience in many research (including 17 EU-funded) projects. $\mathrm{He}$ received his Diploma in Electrical \& Computer Engineering (2003) and his MBA in TechnoEconomics (2005) from National Technical University of Athens (NTUA). In 2011, he received his $\mathrm{PhD}$ on Software Radios, Cognitive Radio, and Adaptive Wireless (NTUA). He is the co-founder of Feron Technologies (2015), a telecom SME, where he leads R\&D activities. His interests include Vehicular Communications, Adaptive PHY, MIMO systems, and Channel Modeling. He has more than 35 publications in journals, conferences, and book chapters.

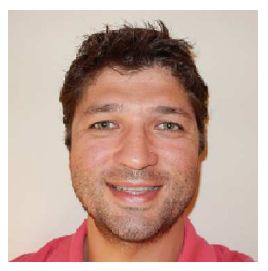

Fotis Foukalas received his 5 years Diploma (M.Eng.) in Electrical and Computer Engineering from the Aristotelian University of Thessaloniki, Greece in 2001 and his MSc in ICT from National Technical University of Athens in 2004. He received also his Ph.D. in Informatics and Telecommunications from the National and Kapodistrian University of Athens (University of Athens) in 2011. His research interests are in the area of wireless mobile networks. He was the coordinator of the SOLDER FP7 ICT project on Future Networks and co-principal investigator of the p-Sparc NPRP QNRF project from Nov. 2013 to Nov.2016. He is now a senior researcher at IoT Research Center at Technical University of Denmark. 